\title{
A heavily subtituted manganite in an ordered nanocomposite for long-term energy applications
}

\section{Federico Baiutti}

Catalonia Institute for Energy Research - IREC https://orcid.org/0000-0001-9664-2486

\section{Francesco Chiabrera}

Catalonia Institute for Energy Research - IREC https://orcid.org/0000-0001-8940-2708

\section{Matias Acosta}

University of Cambridge https://orcid.org/0000-0001-9504-883X

\section{David Diercks}

Colorado School of Mines https://orcid.org/0000-0002-5138-0168

\section{David Parfitt}

Coventry University

José Santiso

ICN2 (Institut Catala de Nanociencia i Nanotecnologia) https://orcid.org/0000-0003-4274-2101

\section{Xuejing Wang}

Purdue University

\section{Alex Morata}

Institut de Recerca de l'Energia de Catalunya

\section{Andrea Cavallaro}

Imperial College London https://orcid.org/0000-0002-6688-1643

\section{Haiyan Wang}

Purdue University West Lafayette https://orcid.org/0000-0002-7397-1209

\section{Alexander Chroneos}

Imperial College London

Judith MacManus-Driscoll

University of Cambridge https://orcid.org/0000-0003-4987-6620

Albert Tarancón ( $\nabla$ atarancon@irec.cat )

Institut de Recerca de l'Energia de Catalunya https://orcid.org/0000-0002-1933-2406

\section{Article}

Keywords: nano-engineered composite oxides, energy materials, nanocomposites

Posted Date: January 12th, 2021 
DOl: https://doi.org/10.21203/rs.3.rs-134793/v1

License: (c) (1) This work is licensed under a Creative Commons Attribution 4.0 International License. Read Full License

Version of Record: A version of this preprint was published at Nature Communications on May 11th, 2021. See the published version at https://doi.org/10.1038/s41467-021-22916-4. 


\section{A heavily substituted manganite in an ordered nanocomposite for}

\section{long-term energy applications}

F. Baiutti (1), * F. Chiabrera (1), M. Acosta (2), D. Diercks (3), D. Parfitt (4), J. Santiso (5), X. Wang

(6), A. Cavallaro (7), A Morata (1), H. Wang (6), A. Chroneos (4), J. MacManus-Driscoll (2), A.

Tarancon $(1,8) *$

(1) Catalonia Institute for Energy Research (IREC), Jardins de Les Dones de Negre 1, 08930 Sant Adrià del Besòs, Barcelona, Spain

(2) Department of Materials Science and Metallurgy, University of Cambridge, 27 Charles Babbage Road, Cambridge CB3 0FS, United Kingdom

(3) Department of Metallurgical and Materials Engineering, Colorado School of Mines, Golden, CO 80401, United States

(4) Faculty of Engineering, Environment and Computing, Coventry University, Priory Street, Coventry CV1 5FB, United Kingdom

(5) Catalan Institute of Nanoscience and Nanotechnology, ICN2, CSIC and The Barcelona Institute of Science and Technology (BIST), Campus UAB, 08193 Bellaterra, Barcelona.

(6) School of Materials Engineering, Purdue University, West Lafayette, Indiana 47907, United States

(7) Department of Materials, Imperial College London, Prince Consort Road, London SW7 2BP, United Kingdom

(8) ICREA, 23 Passeig Lluís Companys, Barcelona 08010, Spain.

*Corresponding authors: atarancon@,irec.cat, fbaiutti@irec.cat 


\begin{abstract}
The implementation of nano-engineered composite oxides opens up the way towards the development of a novel class of superior energy materials. Vertically aligned nanocomposites are characterized by a coherent, dense array of vertical interfaces, which allows for the extension of local effects to the whole volume of the material. Here, we use such a unique architecture to fabricate highly electrochemically active nanocomposites of lanthanum strontium manganite and doped ceria with unprecedented stability and straight applicability as functional layers in solid state energy devices. Direct evidence of synergistic local effects for enhancing the electrochemical performance, stemming from the highly ordered phase alternation, is given here for the first time using atom-probe tomography combined with oxygen isotopic exchange. Interface-induced cationic substitution, enabling lattice stabilization, is presented as the origin of the observed long-term stability. These findings reveal a novel route for materials nano-engineering based on the coexistence between local disorder and long-range arrangement.
\end{abstract}

\title{
Introduction
}

Solid Oxide Cells (SOCs), which are capable of reversibly converting chemical to electrical energy with high theoretical efficiency and a minimized footprint, ${ }^{1,2}$ can play a pivotal role in the widespread implementation of zero-emission next-generation portable energy devices. By means of modern fabrication technologies, SOCs can take advantage of beyond-state-of-the-art concepts for materials' nanoengineering, this way achieving enhanced performance and further downscaling. ${ }^{3}$ To date, however, sluggish electrode catalytic activity, poor thermochemical stability, ${ }^{4}$ and limitations in the effective implementation of advanced nanoscale materials in real devices, have hindered the scalability of SOCs technology and its viability as a portable energy source. ${ }^{5}$ In order to improve the performance of SOC air electrode without using expensive and rare noble metals, the implementation of functional ceramic nanostructures based on mixed ionic-electronic conducting oxides (MIEC) represents a promising 
approach. ${ }^{6}$ In MIEC materials (e.g. doped perovskite manganites, cobaltites, ferrites), a good trade-off between the free energy of the oxygen redox cycle and the enthalpy of oxide reduction can be achieved, ensuring high electrocatalytic activity on the whole free surface together with fast oxygen ion transport. ${ }^{7}$ Most importantly, when MIECs are employed in the form of thin films, the dense microstructure determines excellent in-plane percolation, while the possibility of nanostructuring via cutting-edge thin film techniques opens up the path towards the exploitation of nanoscale effects such as local fast oxygen reduction kinetics and transport. ${ }^{8,9}$ Although the implementation of MIEC-based thin films for the fabrication of SOC functional layers is of great potential e.g. for avoiding the occurrence of areas with high electronic current density or high oxygen chemical potential which may potentially lead to device failure, ${ }^{10}$ intrinsic limitations still occur especially in relation to a poor thermal stability owing to dopant segregation towards the surface. Limiting cationic migration is of paramount importance in order to avoid the possible formation of insulating secondary phases and to maintain the surface activity over time. ${ }^{11,12}$ State-of-the-art strategies for enhancing MIEC thin film performance comprise strain engineering via epitaxial growth, ${ }^{13,14}$ chemical doping ${ }^{15}$, introduction of an interface (so-called heterogeneous doping), ${ }^{9,16-}$ ${ }^{18}$ or surface decoration. ${ }^{19}$ Such methods, however, are often limited to model systems with no technological applicability, and very few of them have proven to be successful in simultaneously improving the initial ORR kinetics while mitigating detrimental surface cationic segregation. ${ }^{20}$

In the present work, we describe the fabrication of a self-assembled thin film nanocomposite that takes advantage of an ideal off-plane architecture, extended all along the layer, to accelerate the solid-gas reaction kinetics and charge transport, while blocking thermal degradation. The structure combines an electronic conductor with a good electrocatalytic performance such as $\mathrm{La}_{0.8} \mathrm{Sr}_{0.2} \mathrm{MnO}_{3}$ (LSM) with a fast ionic conductor, namely $\mathrm{Ce}_{0.8} \mathrm{Sm}_{0.2} \mathrm{O}_{2}$ (SDC), ${ }^{21}$ in a vertically aligned, nanoscale phase alternated, fashion (vertically aligned nanostructures - VANs). ${ }^{22}$ The fabrication of such VANs is based on a single-stage self-assembling thin film process and uses an yttria-stabilized zirconia (YSZ) electrolyte as a support. We stress here that such a support is considered as an ideal platform for the widespread implementation of 
functional oxide nanomaterials in devices. ${ }^{23}$ The obtained ordered nanostructure is also ideal for fundamental investigations on oxygen reduction reaction (ORR) kinetics. Direct evidence of a synergistic mechanisms for oxygen incorporation and mass transport at the nanoscale has been obtained by a novel technique based on isotopic oxygen labelling combined with atom-probe tomography (APT), achieving unique nm-resolution for describing the oxygen incorporation mechanism. Finally, theoretical calculations allowed us to directly link the observed high thermal stability to the local chemical environment in the LSM columns, pointing out the relevance of self-driven substitutional doping for the final long-term functionalities.

\section{Results and discussion}

Nano-engineered VAN structures have been extensively investigated in the last years. Model systems, generally characterized by 'nanocolumn in matrix' mesostructures (domain-matching epitaxy), ${ }^{24,25}$ have revealed unexpected properties including e.g. enhanced ionic conductivity ${ }^{26}$ controlled resistive switching ${ }^{27}$ and improved photocatalytic activity. ${ }^{28}$ Such functionalities have been ascribed to the entanglement of interface effects including elastic strain and ionic or electronic redistribution. ${ }^{29}$ Some reports also deal with effective nanocomposite layers or interlayers for electrochemical applications: Yoon et al. and Cho et al. proposed similar solutions, based on self-assembled MIEC-fluorite thin film vertical nanocomposites, for application as functional layers in fuel cells,${ }^{30}$ while Ma et al. studied the fundamental properties of Co-based vertically aligned nanostructures, highlighting enhanced ORR kinetics. ${ }^{31}$ Although such works demonstrate the great potential of the approach, to the best of the authors' knowledge, so far inert model substrates had to be employed for the realization of ideal structures, while the use of "non-ideal" supports such as electrolyte layers irremediably led to the formation of arrangements with higher disorder (i.e. incoherent interfaces and low interface density), possibly inhibiting the full exploitations of VANs in real electrochemical devices. Here instead, we 
succeeded in fabricating LSM-SDC VAN structures by pulsed laser deposition (PLD) directly on highly oriented single crystal YSZ, possessing excellent out-of-plane epitaxy, maximized interface density $\left(>10^{6}\right.$ $\mathrm{cm}^{\mathrm{cm}} \mathrm{cm}^{2}$ ) and long-range order. This results in the combination of key features for SOC functional layer applications: remarkable ORR kinetics and, most importantly, high stability against temperature-driven cationic migration. A sketch of the LSM-SDC VAN mesostructure, composed by LSM pillars in an SDC continuous matrix, is depicted in Figure 1 (panel a), together scanning transmission electron microscopy (STEM - panels b to d) and high-resolution x-ray diffraction (XRD - panel e). In the lower-magnification high-angle angular dark field (HAADF) STEM image of a thin cross-sectional lamella (Fig. 1b), one can appreciate the thin columnar, long-range ordered, mesostructure for large portions of the film (column width is as narrow as $\approx 5 \mathrm{~nm}$, length $\approx 100 \mathrm{~nm}$, no tortuosity). One can also see that no porosity is present. The precise sequence of alternating ( $\mathrm{La}, \mathrm{Sr}, \mathrm{Mn}$ )- and Ce-based phases can be clearly identified in Fig. 1c, where a HAADF image and an Energy-dispersive X-ray spectroscopy (EDS) color maps of the area highlighted in Fig. 1a are shown. In the HAADF image, owing to the higher $Z$ numbers of Ce and Sm, SDC columns have higher contrast than those of LSM. Interestingly, the color map underlines not only the clear separation between the LSM columns (dominated by Sr, Mn) and the SDC matrix (Ce, Sm), but also a certain degree of cationic intermixing especially between $\mathrm{La}, \mathrm{Sm}$ and Ce. A quantitative and theoretical analysis on cationic redistribution will be discussed in detail in later paragraphs. The HRTEM of a representative columnar region is shown in Fig. 1d, where one can notice a perfect local order. The clear atomic arrangement can be further analyzed in Fig. 1e (inverse filtered fast Fourier transform (FFT) of the region in Fig. 1d): A structurally coherent, relationship is established out-of-plane (zdirection) between the two phases. Also, no evidence of other phases or of spurious orientations are detected in these regions. The in-plane and out-of-plane orientation relationships between the film phases and the substrate in such highly ordered majority portions are LSM (001) || SDC (100) || YSZ (100) and LSM (110) || SDC (001) || YSZ (001), respectively, as sketched in the bottom-left inset of panel d. A dedicated X-Ray diffraction analysis by means of reciprocal space mapping (XRD-RSM) of the matching planes in the $z$-direction i.e. (110) for LSM (110-LSM) and (001) for SDC (001-SDC) is shown in Fig. 
1ef. Here, the perfect alignment of the LSM and SDC reflection in $z$ confirms the perfect lattice match in the out-of-plane direction at the global scale. Interestingly, the resulting lattice spacing for LSM $\left(d_{\mathrm{x}}\right.$ $=3.874 \AA$ and $d_{\mathrm{z}}=2.739 \AA$ for the in-plane and for the $z$-direction, respectively, vs $d=3.906 \AA$ for bulk LSM in pseudocubic notation) indicates an isotropic compressive deformation of the cell $(-0.82 \%)$.

(Please note here that $d_{\mathrm{z}}$ refers to the (110) direction and the corresponding out-of-plane lattice parameter is $d_{z} \cdot \sqrt{2}=3.874 \AA$.) The reduced lattice parameter is consistent with cationic substitution (Ce and $\mathrm{Sm}$ replacing La - cf. Figs. 1b), in agreement with previous reports and as also confirmed by the simulations presented later in Fig. 5. ${ }^{22}$ Please refer to Supplementary Note 1 for the complete XRD characterization of the films.
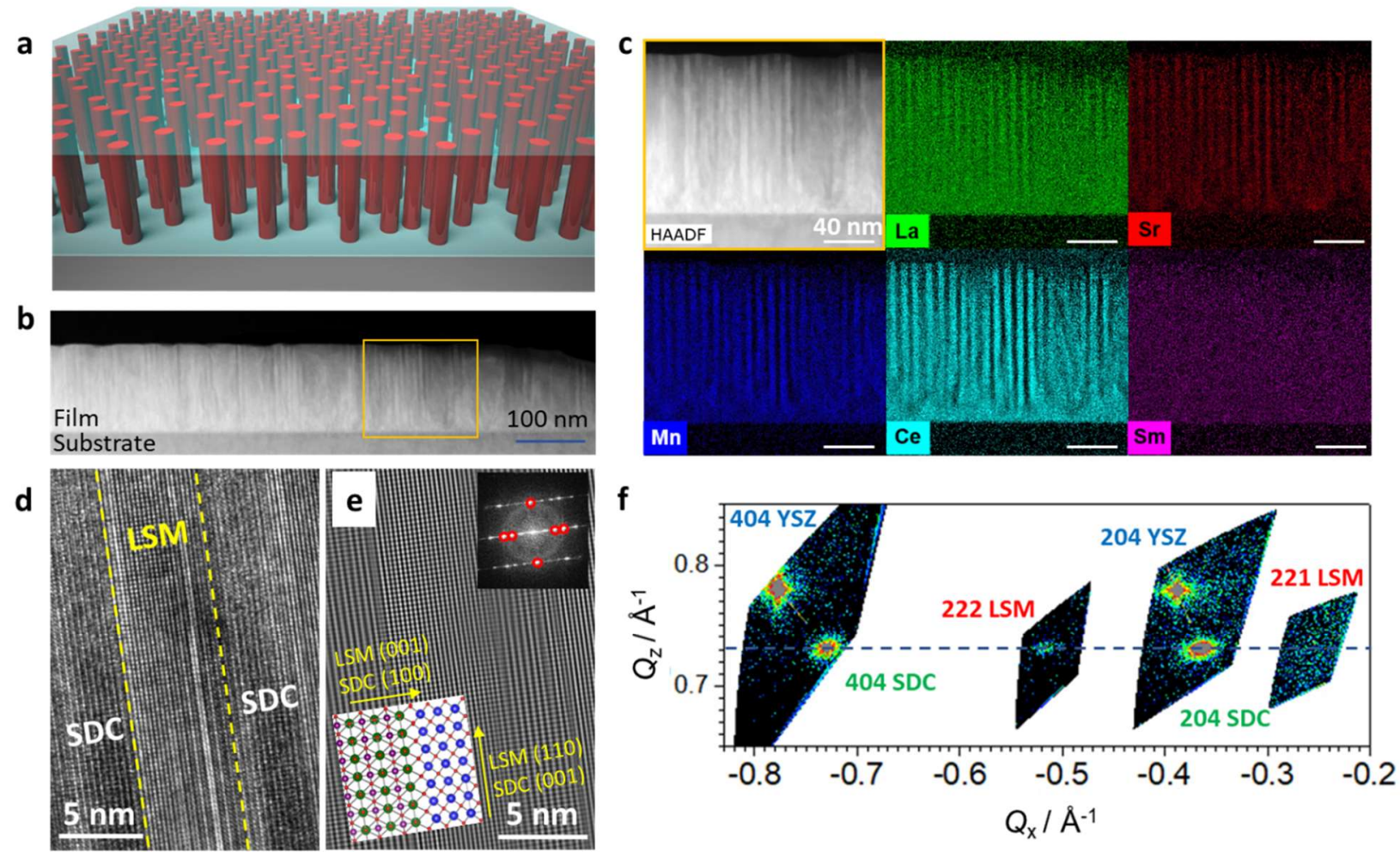

Figure 1. Structural investigation of LSM-SDC. a) Sketch of the LSM-SDC VAN structure. b) Lowmagnification image of the film, highlighting the long-range order of the pillar structure. The thickness decrease on the right side should be ascribed to TEM lamella preparation. c) HAADF image and EDS 
colour maps for a representative columnar region of the film d) HR-TEM of the alternating SDC and LSM columnar structure. e) IFFT of the region highlighted in red in panel c. The atomic ordering and the epitaxial relationship between the LSM (out-of-plane [110], zone axis [1-10]) and the SDC (out-of-plane [001], zone axis [100]) regions is clearly visible, as schematically sketched by the bottom inset. In the inset of top, the original FFT and the filtered reflections (highlighted by the red circles) are shown.f) RSM of the out-of-plane matching 001-SDC ((404) and (204) SDC reflections) and (110)-LSM (222) and (221) reflections) domains. The (404) and (202) substrate reflections are also visible. The dotted horizontal line passes through the maxima of the LSM and SDC reflections, highlighting perfect out-ofplane lattice match between the two phases. Please note that the structural investigation was carried out after after thermal ageing $\left(700^{\circ} \mathrm{C}, 100 \mathrm{hrs}\right)$.

Complementary to HR-TEM, the use of Atom Probe Tomography (APT) allowed us to obtain meaningful insights on the structure chemistry and on the oxygen kinetics, as summarized in Figure 2. The technique, which is based on the Time-of-Flight (ToF) analysis of atoms evaporated through a pulsed laser impinging a highly biased sample tip, possesses unique capabilities for 3D imaging and precise quantitative chemical composition analysis with sub-nm resolution. ${ }^{32}$ In Figs. $2 \mathrm{a}$ and $2 \mathrm{~b}$, projections of the spatial distribution of Mn (red - representative of LSM) and Ce (light blue - for SDC) over a sample tip (HAADF-STEM in Supplementary Figure 2) are reported. Consistently with the TEM analysis (cf. Fig. 1), one can observe the presence of well-defined LSM pillars, immersed in an SDC matrix. The composition of the two phases has been carefully analyzed along a region crossing multiple LSM and SDC domains (blue cylinder in Figs. 2a and 2b). The resulting elemental distribution is reported in Fig. 2c and clearly confirms the modulation of $\mathrm{Mn}, \mathrm{Sr}$ and $\mathrm{Ce}$ when crossing each phase. The non-negligible intermixing of the other cations is clear (cf. also Fig. 1b) and results in the substitution of La by Ce and Sm in LSM (12.3 \pm 1.3 and $3.8 \pm 0.5$ at. \%, respectively), while a large La content is found in SDC (6.7 \pm 0.9 at. \%). The measured elemental atomic fraction is reported in Table 1. Please refer Supplementary Note 2 for more details on the method and for Supplementary Figure 3 for the cationic spatial distribution. 
While such a high level of intermixing is expected to have only a limited impact on the oxygen mobility in SDC owing to dopant excess $(\approx 32$ at. $\% \mathrm{Sm}+\mathrm{La}),{ }^{33}$ the replacement of La in LSM by Ce and Sm plays a key role, according to our theoretical calculations, in the oustanding thermal stability of the structure as discussed later (cf. Fig. 45).

Table 1. Elemental atomic fractions in LSM and SDC. These were calculated dividing the phases into 30 regions and taking as errors the standard deviations of the compositions from each of the individual regions.

\begin{tabular}{|l|r|r|r|r|r|r|}
\hline \multicolumn{2}{|l|}{ LSM overall composition } & & \multicolumn{3}{l|}{ SDC overall composition } \\
\hline & Measured cts. & at. \% & Nominal \% & Measured cts. & at $\%$ & \multicolumn{2}{l|}{$\begin{array}{l}\text { Nominal } \\
\%\end{array}$} \\
\hline Mn & 26463.92 & $15.8 \pm 1.6 \%$ & 20 & 13066.19 & $3.6 \pm 1.8 \%$ & 0 \\
\hline $\mathbf{C e}$ & 20543.8 & $12.3 \pm 1.3 \%$ & 0 & 79921.37 & $22.0 \pm 1.1 \%$ & 27.5 \\
\hline $\mathbf{L a}$ & 16264.88 & $9.7 \pm \%$ & 16 & 24408.18 & $6.7 \pm 0.9 \%$ & 0 \\
\hline $\mathbf{S r}$ & 4386.603 & $2.6 \pm 0.4 \%$ & 4 & 1680.473 & $0.5 \pm 0.3 \%$ & 0 \\
\hline $\mathbf{S m}$ & 6444.194 & $3.8 \pm 0.5 \%$ & 0 & 12666.99 & $3.5 \pm 0.7 \%$ & 6.9 \\
\hline${ }^{\mathbf{1 8}} \mathbf{O}+{ }^{\mathbf{1 6}} \mathbf{O}$ & 93463.57 & $55.8 \pm 1.7 \%$ & 60 & 231736.7 & $63.8 \pm 2.2 \%$ & 65.6 \\
\hline
\end{tabular}

Taking advantage of the highly ordered and dense microstructure of our VANs, we employed APT for providing an unprecedented analyisis of the electrochemical functionalites of the VAN functional layer at the nanoscale (oxygen mass transport properties) via direct quantification of oxygen diffusion profiles in VANs exchanged with oxygen isotopes $\left({ }^{18} \mathrm{O}\right)$ under controlled conditions of temperature and time..$^{34,35}$ Unlike conventional Isotope Exchange Depth Profiling (IEDP) by Time-of-Flight Secondary Ion Mass Spectrometry (ToF-SIMS), ${ }^{36}$ which is commonly employed in bulk and homogeneous materials, our approach is characterized by a greatly enhanced lateral resolution down to the nm-level (vs $>100 \mathrm{~nm}$ for IEDP). ${ }^{37}$ Fig. $2 \mathrm{~d}$ represents a snapshot of the oxygen incorporation in a LSM-SDC VAN after $6000 \mathrm{~s}$ of exchange with labelled oxygen at $T=550^{\circ} \mathrm{C}$, showing concentration maps of different cations and oxygen atoms as obtained from APT. In the top panels, one can immediately recognize the LSM pillars, indicated by the presence of high Mn concentration areas, surrounded by a ceria matrix. In the bottom panels, areas 
of maximum ${ }^{18} \mathrm{O}$ concentration, corresponding to oxygen incorporated during the exchange, are clearly correlated to the SDC matrix while LSM pillars present much lower concentration. This indicates that oxygen prefers to diffuse along the nm-wide ceria regions, in its way to the electrolyte, after the incorporation through the active surface. Moreover, an ${ }^{18} \mathrm{O}$ concentration decay profile is clearly visible in LSM (maximum concentration at the LSM-SDC interface), as a direct consequence of lateral oxygen diffusion from the SDC, while vertical transport in LSM is negligible (cf. also Supplementary Note 3). The VAN combines the excellent ionic conductivity of SDC with the great oxygen affinity of the LSM (typically hindered by its poor oxygen diffusivity) resulting in a perfect synergy that provides an ideal electrode material by design. ${ }^{38}$ This job-sharing effect is further enhanced by the unique VAN microstructure, which is characterized by no tortuosity for mass transport and by maximized triple-phase boundary density $\left(>10^{6} \mathrm{~cm} \cdot \mathrm{cm}^{-2}\right.$, cf. Fig. 1) for oxygen incorporation. Such a precise direct observation of the oxygen kinetics, which is demonstrated here for the first time in the case of VANs, has wide applicability and represents a decisive step forward to the local analysis of oxygen incorporation in nanomaterials, for which singularities are often found at the nm-level (e.g. fast grain boundary oxygen diffusion).$^{38}$ In order to quantify this qualitative picture, the measured diffusion profiles (integrated over the area between the dotted lines in Fig. 2d) were fitted using Finite Element Method (FEM) simulations on a modelled geometry (details on the modelling are given in ef. Supplementary Note 3). The comparison between the measured and the fitted $\left({ }^{18} \mathrm{O} /\left({ }^{18} \mathrm{O}+{ }^{16} \mathrm{O}\right)\right)$ profiles are represented in Fig. 2e. The fitting of the experimental profiles allowed extraction of the oxygen surface exchange coefficient of the composite $\left(k^{*}=2.4 \cdot 10^{-9} \mathrm{~cm} \cdot \mathrm{s}^{-1}\right)$ and the diffusion coefficient of $\operatorname{LSM}\left(D^{*}=3.6 \cdot 10^{-17} \mathrm{~cm}^{2} \cdot \mathrm{s}^{-1}\right)$ at $550{ }^{\circ} \mathrm{C}$ (note that no information on the ion diffusivity is accessible by tracer method for SDC since these regions saturate owing to the very fast transport kinetics). Similar results on a different $2 \mathrm{D}$ cross-section are reported in Supplementary Figure 4. The resulting surface exchange coefficient of the VAN is remarkably higher than the one of $\operatorname{LSM}\left(k^{*} \sim 1 * 10^{-11} \mathrm{~cm}^{2 *} \mathrm{~s}^{-1}\right)$ and also than the one reported for related LSM-YSZ dense composites $\left(k^{*} \sim 2 * 10^{-10} \mathrm{~cm}^{2 *} \mathrm{~s}^{-1}\right)$ at the same temperature. ${ }^{39,40}$ As far as the diffusion coefficient of 
LSM is concerned, this is fully consistent electrochemical measurements by impedance spectroscopy $\left(D^{*}\right.$ impedance $\left.=5.4 \cdot 10^{-17} \mathrm{~cm}^{2} \cdot \mathrm{s}^{-1}\right)$ and with literature data $(\mathrm{cf}$. Supplementary Figure 8$)$.
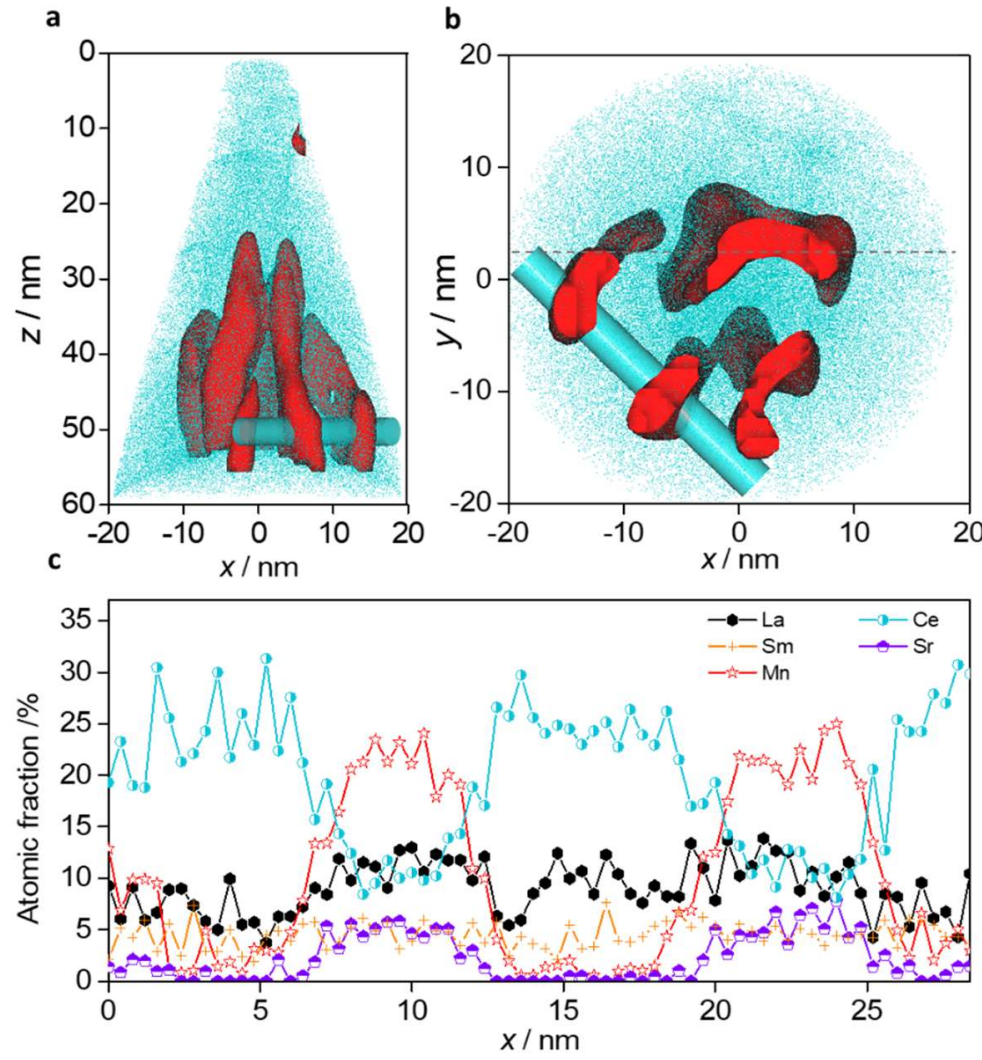

$$
\frac{E}{N}
$$
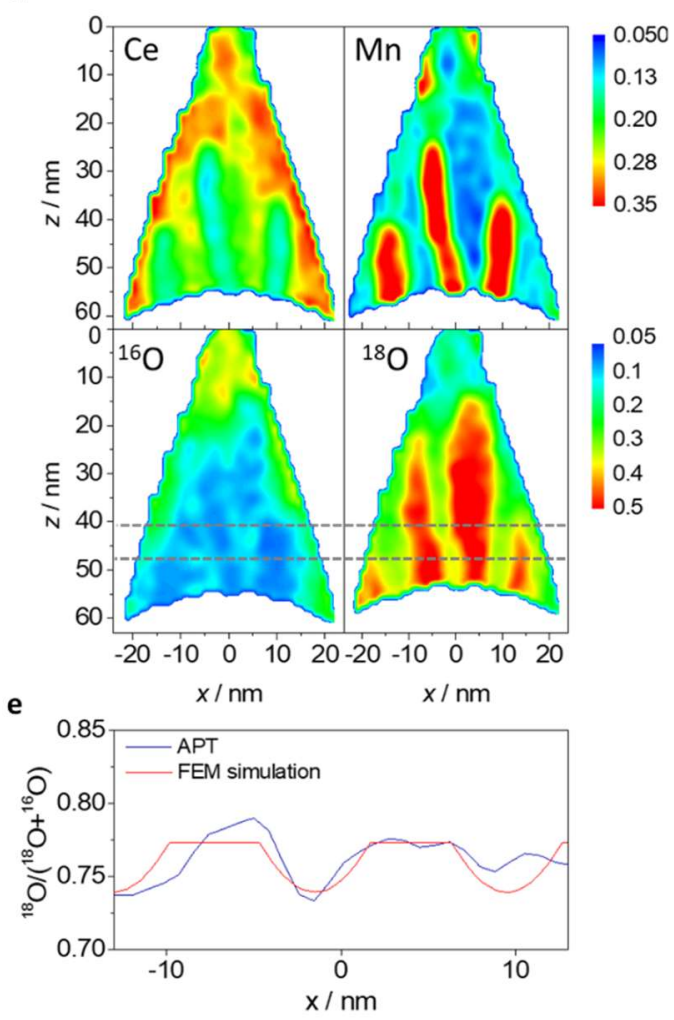

Figure 2. APT on LSM-SDC. Lateral (a) and bottom view (b) 3D renderings, highlighting the areas of LSM (red surface) and SDC (light blue dots) using a 30 at\% La+Sr+Mn isoconcentration surface. c) Cationic atomic fraction along the linescan indicated by the blue cylinder in panels (a) and (b). d) Concentration color maps for $\mathrm{Ce}, \mathrm{Mn},{ }^{18} \mathrm{O}$ and ${ }^{16} \mathrm{O}$ from the slice indicated by the grey dotted line in panel (b). e) Experimental (blue - obtained upon integration of the area between the dotted lines in panel d) and simulated (red) ${ }^{18} \mathrm{O}$ fraction profiles.

Complementary electrochemical characterization was carried out by impedance spectroscopy (EIS) in conventional two-electrode supported electrolyte cells (Au-LSM-SDC VAN/YSZ/Ag) and is summarized in Figure 3. 

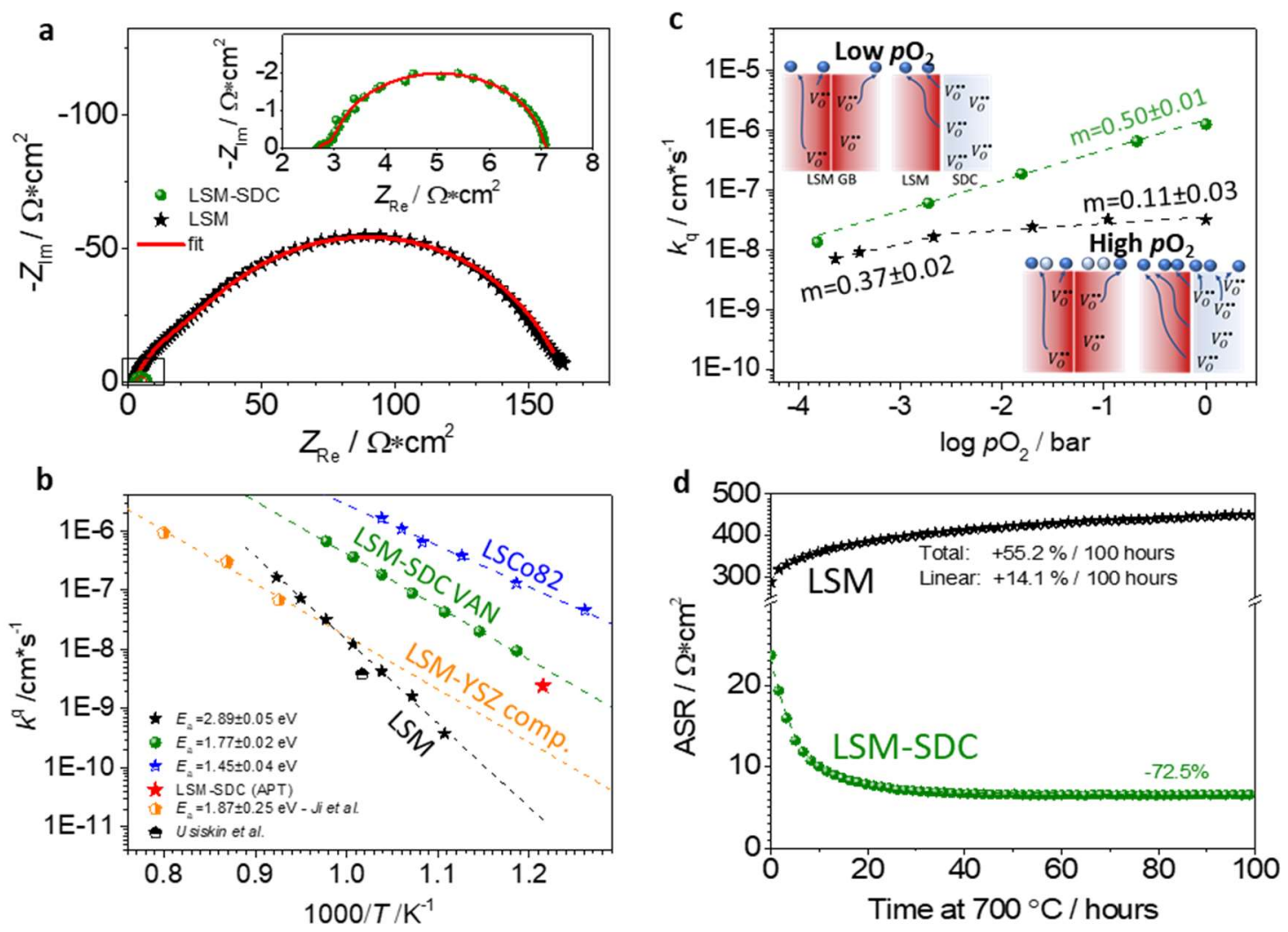

Figure 3. Electrochemical performance of LSM (black stars) and LSM-SDC (green bullets) nanocomposites. a) Representative EIS Nyquist plots measured at $750{ }^{\circ} \mathrm{C}$ (air atmosphere) in an out-ofplane configuration and using a porous Ag layer as a low impedance counter-electrode for LSM and LSM-SDC. The inset shows the LSM-SDC impedance arc on a smaller scale. b) Surface exchange coefficient $k^{q}$ as a function of inverse temperature, as compared to stoichiometric LSM (from our labs and from Ref. ${ }^{40}$ ), state-of-the-art LSM-YSZ dense composites (from Ref. ${ }^{39}$ ) and to state-of-the-art $\mathrm{La}_{0.8} \mathrm{Sr}_{0.2} \mathrm{CoO}_{3}$ (our labs). The red star represents the value resulting from the FEM simulation of the APT ${ }^{18} \mathrm{O}$ fraction profile. c) $\mathrm{pO}_{2}$ dependence for $k^{q}$. The slopes for LSM and LSM-SDC are indicated. d) ASR vs time for LSM and LSM-SDC, measured during ageing treatment. The lines are intended as a guide to the eye.

In Fig. 3a, exemplary Nyquist plots for LSM-SDC and analogous LSM cells are reported $\left(T=750^{\circ} \mathrm{C}\right.$, air atmosphere). LSM exhibits the typical polarization arc of MIEC materials stemming from a co-limited 
process, i.e. ambipolar diffusion $\left(\approx 45^{\circ}\right.$ straight element at high frequencies $)$ and oxygen reduction reaction (semicircle at low frequencies). ${ }^{41}$ One can appreciate that in LSM-SDC both contributions are drastically reduced (cf. also Supplementary Figure 9 for comparison of the area-specific resistance ASR). The polarization arc exhibits an almost perfect impedance arc - indicating that the oxygen incorporation is limited by the surface reduction reaction only - while the mass transport contribution is completely suppressed. This is fully consistent with the APT characterization of the oxygen kinetics highlighting fast vertical diffusion of oxygen through the SDC phase. The Nyquist plot for single phase SDC cells, exhibiting much higher ASR, is reported for comparison, demonstrating the higher performance of the VANs under consideration, is also reported in Supplementary Figure 10.

By adjusting the EIS spectra of the cells with a physically meaningful equivalent circuit (i.e. a modified Jamnik-Maier circuit for LSM and a simple ZARC element for LSM-SDC - please refer to Supplementary Note 4 for further details on impedance spectroscopy analysis), the effective rate constant for the oxygen reduction reaction $k^{q}$ can be obtained. ${ }^{42}$ The results are reported in Fig. 3b, where LSMSDC is put in direct comparison with i) stoichiometric LSM, ii) LSM-YSZ dense composites and iii) state-of-the-art cobalt-based MIEC for intermediate-temperature electrode applications $\left(\mathrm{La}_{0.8} \mathrm{Sr}_{0.2} \mathrm{CoO}_{3}-\right.$ LSCo82). ${ }^{39,40}$ One can see that the oxygen incorporation kinetics are remarkably enhanced in LSM-SDC with respect to LSM ( $\approx 100$ fold enhancement) and to LSM-YSZ. Besides the activation energy $E_{\mathrm{a}}$ is decreased by more than $1 \mathrm{eV}(\approx 2.9 \mathrm{eV}$ for stoichiometric LSM and $\approx 1.8 \mathrm{eV}$ for LSM-SDC), becoming comparable to LSCo $\left(E_{\mathrm{a}} \approx 1.5 \mathrm{eV}\right)$. Please note that, even though much lower values are expected for porous Co-based bulk electrodes, ${ }^{43}$ here the relevant comparison is between dense thin films with potential application as functional layers. In Fig. 3b, also the $k$ value obtained by the FET simulation of the APT oxygen isotope profiles is reported (red star), demonstrating the very good agreement with the impedance data. With knowledge on the elementary processes of oxygen incorporation in LSM (atomic adsorbate $\left(O_{a d}^{-}\right)$incorporation-limited ORR process $),{ }^{41,44}$ it is also possible to provide a mechanistic interpretation of the synergistic LSM-SDC incorporation mechanism by analyzing the oxygen partial 
pressure $\left(p \mathrm{O}_{2}\right)$ dependence of $k^{\mathrm{q}}$. Fig. 3c shows that in the low- $p \mathrm{O}_{2}$ regime, both the single phase material and the nanocomposite follow the expected $\approx 1 / 2$ slope resulting from the final incorporation step (i.e. the encounter between $O_{a d}^{-}$and a lattice oxygen vacancy $\left(V_{O}^{*}\right)$, leading to a predicted reaction rate $R\left(p \mathrm{O}_{2}\right)$ $\propto \theta\left(O_{a d}^{-}\right) \propto p 0_{2}^{\frac{1}{2}}$, where $\theta$ is the surface coverage) being limiting in both cases. Under such conditions, also the absolute $k^{\mathrm{q}}$ values are similar between the single-phase material and nanocomposite, further corroborating this interpretation. However, at high $p \mathrm{O}_{2}$, the limited availability of oxygen vacancies in LSM (which are mostly concentrated at the grain boundaries) governs the saturation in the kinetics of the incorporation process (i.e. sluggish $p \mathrm{O}_{2}-k^{q}$ dependence), resulting in an excess of $O_{a d}^{-}$at the film surface (cf. also Supplementary Figure 911). ${ }^{45}$ Conversely, in the case of LSM-SDC, the supply of oxygen vacancies from SDC allows for readily incorporating oxygen also at high $p \mathrm{O}_{2}$, as schematically depicted in the sketches in Fig. 3c. This demonstrates the beneficial effect of the vertical phase alternation and maximized triple-phase boundary density in the VAN structure in terms of surface oxygen exchange kinetics. Notably, oxygen incorporation in our VANs may be facilitated also by the extension of the active area for oxygen incorporation also to the SDC surface as a consequence of adsorbates "spill-over" from LSM to SDC. ${ }^{46}$

Lastly, in Fig. 3d we report the results on the thermal stability of the SDC-LSM nanocomposites, evaluated by carrying out a 100 hours treatment at $700{ }^{\circ} \mathrm{C}$ in air, while in-situ measuring the polarization resistance. It is well-known that, under such conditions, LSM and doped perovskite materials in general undergo changes in the surface composition as a consequence of dopant migration from the bulk, causing the formation of insulating Sr-based secondary phases. ${ }^{47,48}$ In our case, single-phase LSM undergoes the expected degradation, which is reflected in a 50\% ASR increase in 100 hrs. Surprisingly, the LSM-SDC nanocomposites are characterized by stable thermal behavior with virtually no degradation over the time span under consideration: Even a 70\% decrease of the ASR is observed before stabilization (Please refer to Supplementary Figure 12 for the time evolution of $D^{\mathrm{q}}$ and $k^{\mathrm{q}}$ for LSM and VANs). In order to rationalize such a remarkable behavior, we first carried out complementary microstructural 
characterization by atomic force microscopy (AFM) and scanning electron microscopy (SEM). The results are shown in Figure 4. In Fig. 4a, the comparison between the AFM micrographs from LSM and LSM-SDC before and after thermal treatment $\left(700^{\circ} \mathrm{C}, 100 \mathrm{hrs}\right)$ highlights that no surface evolution is apparent for LSM-SDC, while secondary phase outgrows are formed on the LSM surface. These are ascribed to Sr-based precipitates as a consequence of massive Sr-migration and are responsible for the severe degradation of the surface reactivity owing to a reduced availability of active sites in LSM. ${ }^{49,50}$ The top-view SEM images for the materials after ageing (LSM and LSM-SDC in panels b and c, respectively), provide information at lower-magnification and highlight the profound difference between the two surface: Namely, deleterious Sr segregation is fully hindered in LSM-SDC. Please note that the bulk microstructural stability of VANs had been already been proven by TEM (cf. Fig. 1).
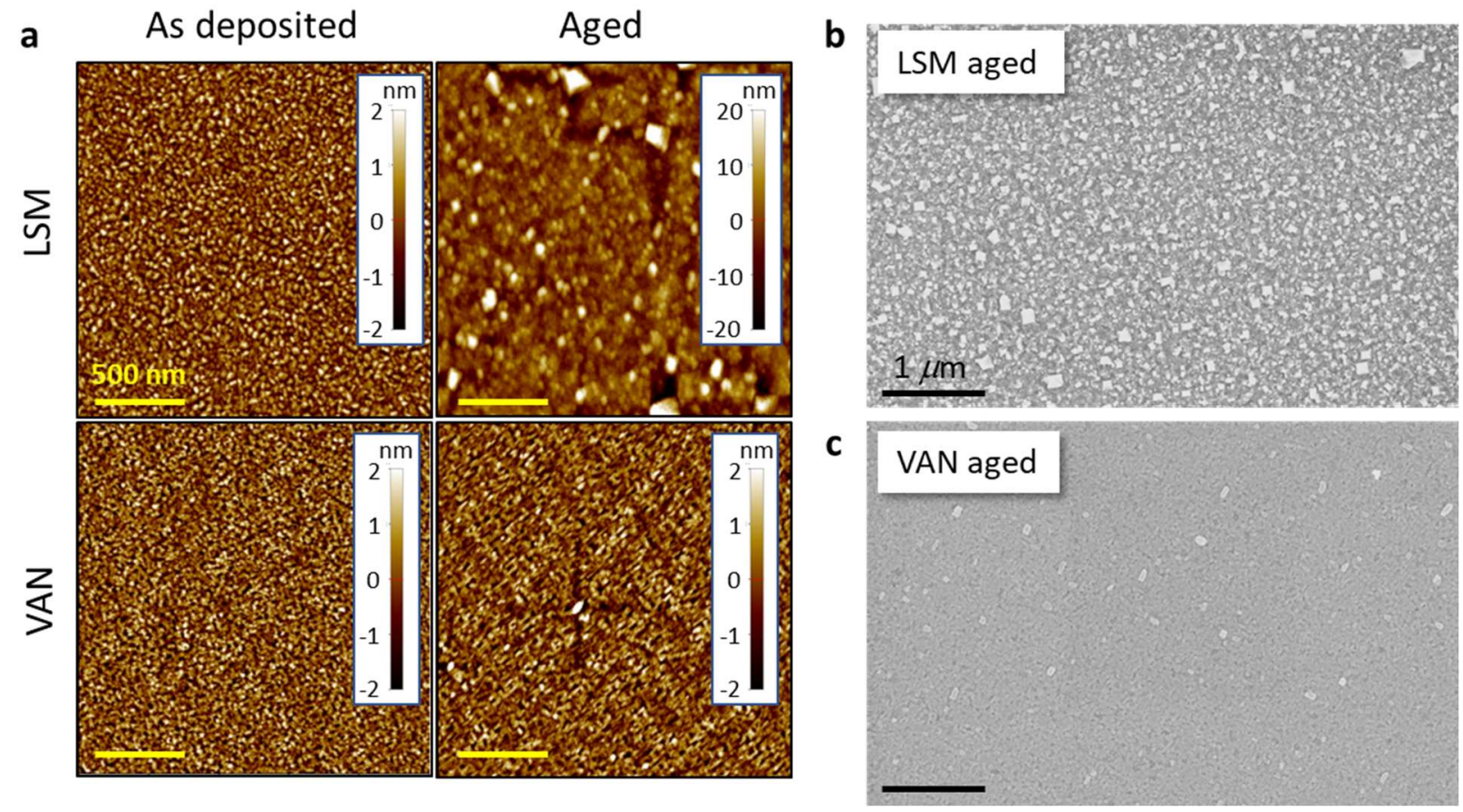

Figure 4. AFM and SEM top-view micrographs for LSM and LSM-SDC. In (a), a full comparison between the surfaces of the two compounds in the as-grown and aged states is provided. The resulting root mean square roughness are: $R_{m s}=0.8$ (7.1) and $R_{m s}=0.7(1.0) \mathrm{nm}$ for LSM-SDC and single phase LSM as 
grown (after ageing), respectively. In panels $(b-L S M)$ and ( $c-V A N)$, lower magnification SEM for the surfaces after thermal treatment (In-Lens detector - $3 \mathrm{kV}$ acceleration voltage).

Such an unexpected behavior for VANs has been quantitatively assessed using state-of-the-art low energy ion scattering (LEIS). LEIS is capable of probing the elemental composition of the very outer atomic surface layer of the material and is therefore well-suited for analyzing surface chemical composition with the highest sensitivity. ${ }^{37}$ The LEIS spectra for VAN as-grown and after thermal ageing (100 hrs at 700 ${ }^{\circ} \mathrm{C}$ ) are reported in Figures 5a and 5b, respectively. The stoichiometric ratios $[\mathrm{Sr}] /[\mathrm{La}]$ can be obtained from the integration of the peak areas. It becomes evident that not just Sr has a very low tendency to segregate in the VAN (the final ratio $[\mathrm{Sr}] /[\mathrm{La}]=0.36 \pm 0.06$ is close the bulk value after the ageing heat treatment - Fig. 5b) but also that the Sr fraction is remarkably decreased in the final state with respect to the material as-grown $([\mathrm{Sr}] /[\mathrm{La}]=0.86 \pm 0.05-$ Fig. $5 \mathrm{a})$. Such a reduction of the $\mathrm{Sr}$ content at the surface fully explains the observed ASR evolution over time, which decreases during the first $\approx 40 \mathrm{hrs}$ and is constant afterwards - cf. Fig 3d, indicating that Sr-La substitution is thermodynamically unfavorable at the VANs surface. Please note the dominant Sr-termination in the as-deposited state is expected to lead to slower ORR kinetics even in the absence of precipitates. ${ }^{51}$ The initial high Sr content for the as-grown material also reveals that the thermodynamic conditions (high $T$ and low $p \mathrm{O}_{2}$ ) and fast kinetics of the film synthesis process (PLD) are very far from the final equilibrium in air, while a bulk-like equilibrium situation is approached upon annealing in the VANs structure. This is in contrast with the common behavior of MIEC materials showing thermally-induced Sr segregation and is combined, in our case, with an enhanced surface kinetics and with non-limiting oxygen diffusion pathways along the SDC matrix. While the effectiveness of the SDC for enhancing ion diffusivity is predictable, the outstanding surface stability of the VAN is surprising and represents an outstanding improvement with respect to state-of-theart MIEC electrodes. 

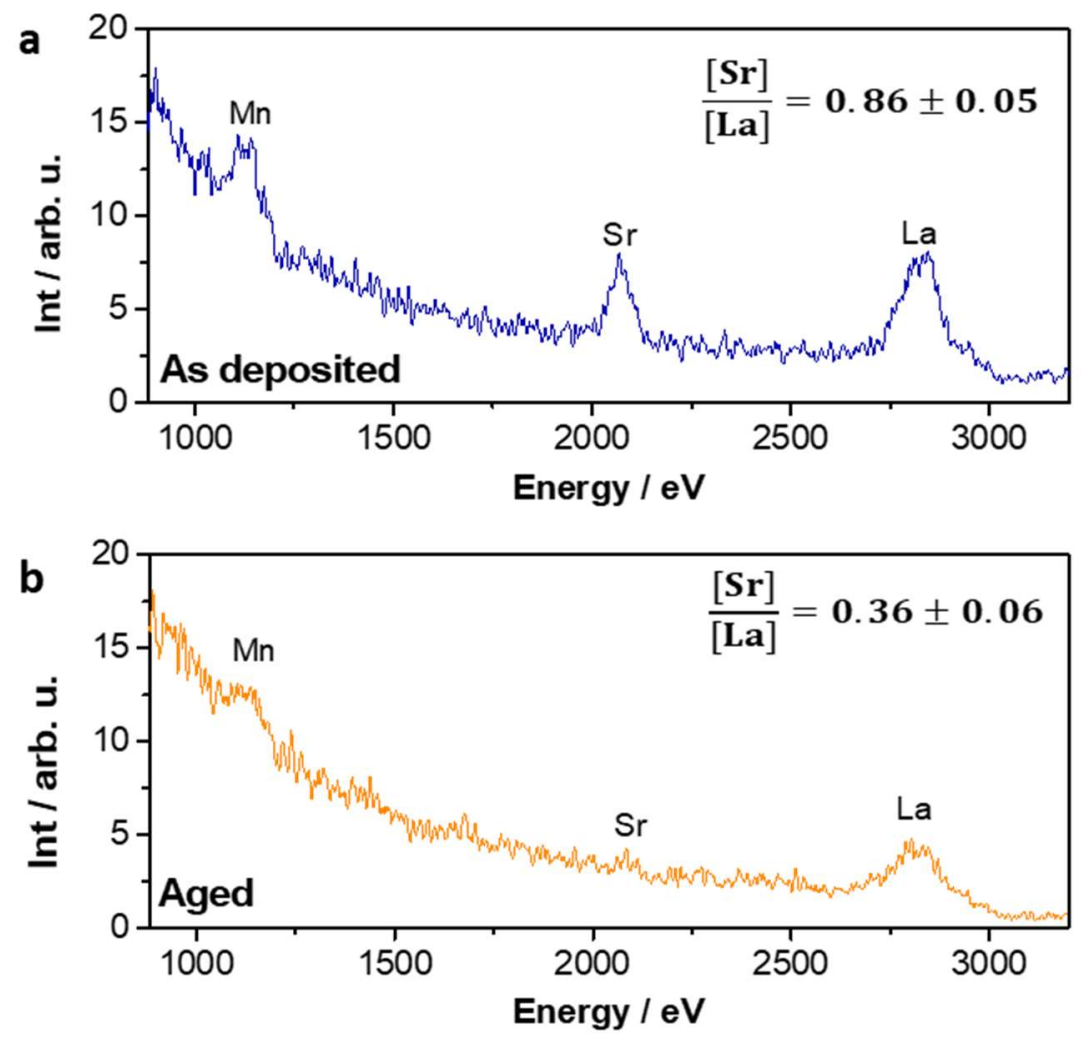

Figure 5. LEIS spectra for LSM-SDC VAN surface as deposited (a) and after thermal treatment (100 hrs, $700{ }^{\circ} \mathrm{C}$ - b). The stoichiometric ratio is obtained from integration of the peak areas.

We performed theoretical calculations based on spin polarized Density Functional Theory (DFT - see further details in Supplementary Note 5 and elsewhere in ref $\left[{ }^{52}\right]$ ) of a cubic perovskite supercell containing 40 atoms (Figure 45a) with the aim of obtaining further insights into the observed thermal stability of the LSM-SDC VAN. This allowed to determine the Sr stability in our LSM structures which, as highlighted experimentally, are characterized by high levels of cation intermixing ( $\mathrm{Sm}$ and $\mathrm{Ce}$ ) and by isotropic compressive strain (cf. Fig. 1). By carrying out energy minimization (Figure 46b), we found that the introduction of Sm cations into the LSM model $\left(\mathrm{La}_{0.75} \mathrm{Sr}_{0.125} \mathrm{Sm}_{0.125} \mathrm{MnO}_{3}\right)$ leads to a pronounced contraction of around $0.3 \%$ while Ce doping, even to quite high levels, has a smaller effect upon the lattice parameter with a drop of $\sim 0.1 \%$ for a composition of $\mathrm{La}_{0.625} \mathrm{Sr}_{0.125} \mathrm{Ce}_{0.25} \mathrm{MnO}_{3}$. Therefore, one can first conclude that the observed isotropic compressive strain of LSM in the VAN structure (cf. Fig. 1) is a 
consequence of the chemical incorporation of Sm from the SDC layers with an additional but smaller influence of Ce at higher dopant contents, ${ }^{53,54}$ ruling out an important role of epitaxial elastic strain. Taking this into account, we directly evaluated the cation interdiffusion as a possible source of the observed Sr segregation suppression. We considered the $\mathrm{Sr}(\mathrm{La})-\mathrm{O}$ polyhedral volume (Figure 6c) and the bond valence sum at each lattice site (Figure 6d) for different cation distributions in order to assess the elastic driving force for atom surface segregation. In bulk LSM with a composition of $\mathrm{La}_{0.875} \mathrm{Sr}_{0.125} \mathrm{MnO}_{3}$, we estimate that the Sr-ion is significantly overbonded with a valence mismatch of $\sim 0.15$ valence units (v.u.), i.e. the surrounding oxygen ions are closer than required by the formal valence state of the $\mathrm{Sr}$ ion, while La ions are slightly underbonded, -0.04 v.u. This opposite valence mismatch is the likely driving force for the exchange of overbonded $\mathrm{Sr}$ ions with underbonded La ions on the surface (in combination with the electrostatic effects). ${ }^{50}$ This is conceptually similar to the Sr ion having a larger ionic radius than the La lattice ion it replaces. ${ }^{48}$ Such an instability is strongly alleviated by cationic intermixing (Figs. 46c and 46d): Particularly, the introduction of Ce leads to an increase in the Sr-O site volume, in turn reducing the $\mathrm{Sr}-\mathrm{O}$ overbonding relative to the La-O polyhedra and thereby decreasing the elastic driving force for Sr surface segregation. In other words, it is the high level of cationic substitution in LSM, leading to the formation of a solid solution with high amounts $\mathrm{Ce}$ (and $\mathrm{Sm}$ ) replacing La, that dictates the excellent long-term behavior of the VAN structure. Critically, the VAN structure under consideration is able to accept Ce concentrations beyond the reported solubility limits of Ce in single phase LSM ( $2 \% \mathrm{~mol}),{ }^{55}$ which is crucial for the observed effects. We assign such an increased solubility limit to a large contribution of surface energy to the final free energy, which is maximized here by the very high interface density; ${ }^{56}$ Notably, within the experimental resolution of the technique, no detectable elemental segregation can be observed by HR-TEM (cf. Fig. 1) or by APT (cf. Fig. 2 and Supplementary Figure 3), confirming the single solid-solution nature of our highly doped LSM in VANs. As a consequence of the observed spontaneous cationic intermixing and interface stabilization, here nominal LSM is, as a matter of fact, a 6 element high entropy oxide having $\mathrm{S}_{\text {config, VAN }}=1.21 R\left(\mathrm{~S}_{\text {config }}=-R \sum_{i=1}^{N} x_{i} \ln x_{i}\right.$ where $x_{i}$ is the atomic fraction in the unit formula as resulting from Table 1). The same calculation for $\mathrm{La}_{0.8} \mathrm{Sr}_{0.2} \mathrm{MnO}_{3}$ 
yields a much lower value $\left(\mathrm{S}_{\text {config, } L S M}=0.56 R\right)$. As resulting from our DFT calculations, such a high level of local cationic intermixing within the LSM phase (high configurational entropy) is key for the oustanding thermal stability of the VAN structure ${ }^{57}$ Because the VAN structure is self-assembled, both the composition and interfaces which form at the growth temperature are the stable ones, i.e. they should not change with further annealing under conditions similar to the growth conditions as they are the energetically preferred. This is very different to the case of artificially- grown superlattice structures, where the artificially-formed interfaces are unlikely to be the equilibrium ones (i.e. coherence is lost above a critical thickness and chemical instabilities may occur during operation).

a

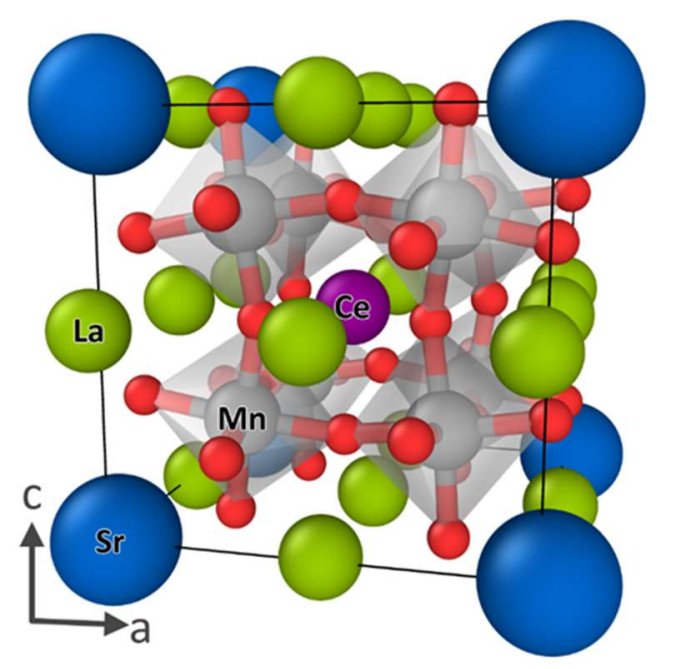

b

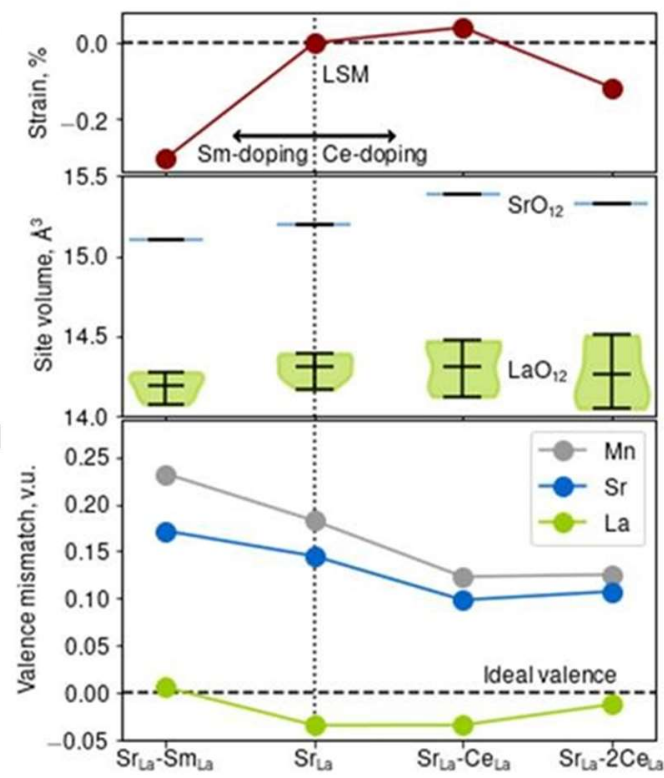

Figure 46. (a) Example of a 40-atom periodic supercell used in DFT calculations showing relaxed positions of the Mn (grey), Sr (blue), La (green), $O$ (red) and Ce (purple) ion and (b) calculated values of the lattice strain due to chemical doping, (c) a box plot showing the mean and distribution of site volumes around the Sr (blue) and La (green) sites and (d) bond valence mismatch at the ion positions for the different cation dopants, where for example $\mathrm{Sr}_{L a}-S m_{L a}$ corresponds to a stoichiometry of $\mathrm{La}_{0.75} \mathrm{Sr}_{0.125} \mathrm{Sm}_{0.125 \mathrm{MnO}}$. 


\section{Conclusions}

In summary, we were able to combine, at the nanoscale, the excellent oxygen exchange properties of LSM with the good diffusivity of SDC to fabricate nearly ideal electrode functional layers for SOCs by design. This was achieved using vertically aligned nanostructures. Most importantly we highlighted that, by the spontaneous formation of a heavily co-doped manganite metastable phase in the VAN (high entropy oxide), a stable compound is formed which hinders Sr-segregation to the surface. This resulted in an outstanding long-term durability at typical operational temperatures of solid oxide electrochemical cells. Such exceptional lattice stability is extended to the whole thin film electrode due to the unique features of VANs, which are able to extend genuine interface effects to the whole volume.

The off-plane geometry, together with the intimate phase alternation and the long-range interface coherence, makes the structure ideal for directly probing solid-gas reactions and oxygen transport. Evidence of the presence of a fast oxygen incorporation pathway at the nanoscale along the SDC phase was presented here for the first time using a novel isotopic exchange depth profiling technique based on atom probe tomography. By this new approach we proved that VAN nanocomposites can solve current kinetic limitations of existing electrodes by a synergistic combination of the functionalities of their individual constituents.

Overall, the described VAN architecture is an easy-to-implement, superior alternative to state-of-the-art functional materials for oxide electrochemistry possessing long-term thermal stability and driving fast oxygen reduction kinetics. Our work represents a decisive step forward in the implementation of advanced thin film technology for high performance electrochemical energy conversion devices and opens up a new path for the development of next-generation functional oxides.

\section{Experimental}


LSM-SDC, LSM and LSCo thin films were deposited in a large-area pulsed laser deposition chamber (PVD Systems - PLD 5000) equipped with a $248 \mathrm{~nm} \mathrm{KrF} \mathrm{excimer} \mathrm{laser} \mathrm{(Lambda} \mathrm{Physics} \mathrm{-} \mathrm{COMPex}$ PRO 205) on YSZ (100) substrate (Crystec $\mathrm{GmbH})$ under the following conditions: temperature $800{ }^{\circ} \mathrm{C}$ $\left(600{ }^{\circ} \mathrm{C}\right.$ for LSCo), oxygen pressure 5 mbar, target-substrate distance $90 \mathrm{~mm}$, laser fluency $1.1 \approx \mathrm{J} \cdot \mathrm{cm}^{-2}$, laser frequency $2 \mathrm{~Hz}$ for LSM-SDC, $10 \mathrm{~Hz}$ for LSM and LSCo. A thin ( $\approx 5 \mathrm{~nm})$ buffer layer of $\mathrm{Ce}_{0.8} \mathrm{Gd}_{0.2} \mathrm{O}_{2}$, fabricated under the same conditions, was deposited between LSCo and YSZ in order to avoid the formation of secondary phases. Since $z$-resolved ${ }^{18} \mathrm{O}$ labelling experiments are only possible for films grown on an inert substrate, a perovskite $\mathrm{SrTiO}_{3}$ substrate $(100$ - Crystec $\mathrm{GmbH})$ was employed for the sample that underwent APT analysis.

LSM-SDC films were fabricated using home-made targets obtained by mixing commercial powders of $\mathrm{La}_{0.8} \mathrm{Sr}_{0.2} \mathrm{MnO}_{3}$ and $\mathrm{Ce}_{0.8} \mathrm{Sm}_{0.2} \mathrm{O}_{2}(1: 1 \mathrm{wt}$ \% - Kceracell $)$ via ball milling in ethanol solution. The dried powder mix was uniaxially pressed ( $7 \mathrm{MPa}, 30 \mathrm{~s}$ ) to form a pellet (diameter $\approx 1$ inch). Sintering was carried out at $1300^{\circ} \mathrm{C}$ for 4 hours (heating and cooling ramps $\approx 5^{\circ} \mathrm{C} / \mathrm{min}$ ). A commercial target was used for LSM and LSCo.

TEM and HAADF STEM imaging, EDS mapping and line scans were acquired by FEI Talos F200X. The TEM samples were prepared using conventional mechanical procedures including polishing, dimpling, and ion milling (PIPS 691). Films were analysed by XRD by using a lab diffractometer with 4angle goniometer (MRD X'Pert Pro from Malvern-Panalytical) and $\mathrm{Cu} \mathrm{K} \alpha$ tube and 2 x Ge(110) monochromator. SEM and AFM characterization were performed in a Zeiss Auriga and a Park System, respectively.

Atom probe tomography (Cameca LEAP 4000X Si) was performed on focused ion beam prepared specimens (FEI Helios NanoLab 600i) that were mounted on TEM grids and hardware that allowed for TEM imaging (FEI Talos F200X) and analysis of the APT specimens. ${ }^{58,59}$ The $355 \mathrm{~nm}$ laser-pulsed APT analysis was performed at $57.3 \mathrm{~K}$ using either a 0.8 or $24 \mathrm{pJ}$ laser energy and $500 \mathrm{kHz}$ pulse rate. The 
flight path length was $90 \mathrm{~mm}$ and the ion detection rate was set to 3.5 ions per 1000 pulses, resulting in a bias range of $2200-5000 \mathrm{~V}$ during the data collection. Reconstructions were generated in Cameca's IVAS 3.6.14 software using the TEM images of the specimens as a guide for setting the reconstruction parameters. Atomic volumes of the species were adjusted based on the unit cell parameters of the oxides. ${ }^{18} \mathrm{O}$ tracer annealing was carried out at a temperature of $550{ }^{\circ} \mathrm{C}$ for $6000 \mathrm{~s}$ in an atmosphere of $200 \mathrm{mbar}$ of enriched oxygen $\left(90 \%{ }^{18} \mathrm{O}\right.$ concentration). Prior to the exchange, an annealing (12 hours) in natural oxygen was carried out.

The electrochemical characterization of the films was carried out using a Novocontrol impedance spectrometer using a frequency range $10^{6}-0.1 \mathrm{~Hz}$ and a voltage amplitude of $0.05 \mathrm{~V}$. A low-impedance Ag counter-electrode was applied by brushing Ag conductive paste (Sigma Aldrich), while a porous Au paste was applied on top of the films to ensure a homogeneous current distribution. A fine gold mesh was used as a current collector on both sides. Measurements were carried out in a ProboStat test station (NorECs) placed inside a vertical furnace and using synthetic air atmosphere. Atomic force microscopy was carried out in non-contact mode using a XE 100 Park System Corp equipment. The images were analyzed using XEI software (Park System Corp.).

FEM simulations were performed by COMSOL Multiphysics using the Transport of diluted species module.

To explore the uppermost sample surface chemical composition, a Qtac100 Low Energy Ion Scattering (LEIS) instrument (ION-TOF GmbH) was employed. Neon primary ion beam was chosen for its higher mass resolution with respect to the elements of LSM-SDC composite analysed in this study. $\mathrm{Ne}^{+} 5 \mathrm{keV}$ ion beam was rastered over a sample area of $500 \mu \mathrm{m}$ x $500 \mu \mathrm{m}$. The energy range was maintained in $850-3600 \mathrm{eV}$. The $\mathrm{Ne}^{+}$dose was kept around $5 \times 10^{14}$ ions $/ \mathrm{cm}^{2}$ for each analysis. Cerium mean isotope LEIS peak overlaps with the lanthanum one. For this reason, the cerium higher mass isotope was also fitted to separate in this way cerium from lanthanum contribution. 


\section{Acknowledgments}

J.S. acknowledges the support of ICN2 (funded by the CERCA programme/Generalitat de Catalunya and by the Severo Ochoa programme SEV-2017-0706) for the XRD measurements. M.A. acknowledges the support from the Feodor Lynen Research Fellowship Program of the Alexander von Humboldt Foundation and the Isaac Newton Trust, 17.25(a). M.A. and J.D. acknowledge the support from the EPSRC Centre of Advanced Materials for Integrated Energy Systems (CAM-IES) under EP/P007767/1. J.D. also acknowledge support from EPSRC grants EP/N004272/1, EP/T012218/1, the Royal Academy of Engineering-CIET1819_24, ERC POC grant 779444, Portapower. X. W. and H. W. acknowledge the support from U.S. National Science Foundation DMR-1565822. This project has received funding from the European Union's Horizon 2020 research and innovation program under grant agreement No 824072 (HARVESTORE) and No 681146 (ULTRASOFC) and was supported by an STSM Grant from the COST Action MP1308: Towards Oxide-Based Electronics (TO-BE), supported by COST (European Cooperation in Science and Technology). The authors thank A. Aguadero for the fruitful discussions and A. Kuzyk for support on graphics.

\section{Author contributions}

Experiments were conceived by F.B., F.C., M.A., J.D., A.T. Sample preparation was carried out by F.B., M. A., electrochemical characterization by F.B. APT was performed by D.D., FET simulations by F.C. DFT calculations were done by D.P. and A.C. XRD analysis was carried out by J.S., TEM analysis by X.W. under the supervision of H.W. A.C. performed the oxygen exchange treatment. F.B. designed the research plan and prepared the initial draft. All the authors contributed to the interpretation and discussion of the experimental data and to editing the initial draft. 


\section{References}

1 E. D. Wachsman and K. T. Lee, Science (80-. )., 2011, 334, 935-939.

2 S. S. Shin, J. H. Kim, K. T. Bae, K.-T. Lee, S. M. Kim, J.-W. Son, M. Choi and H. Kim, Energy Environ. Sci., 2020, 11-16.

3 J. Maier, Nat. Mater., 2005, 4, 805-815.

4 B. C. H. Steele and A. Heinzel, Nature, 2001, 414, 345-352.

5 I. Garbayo, D. Pla, A. Morata, L. Fonseca, N. Sabate and A. Tarancon, Energy Environ. Sci., $2014,7$.

6 Z. Gao, L. V Mogni, E. C. Miller, J. G. Railsback and S. A. Barnett, $1602 \mid$ Energy Environ. Sci, 2016, 9, 1602.

7 W. C. Jung and H. L. Tuller, Adv. Energy Mater., 2011, 1, 1184-1191.

8 M. Acosta, F. Baiutti, A. Tarancón and J. L. MacManus-Driscoll, Adv. Mater. Interfaces, 2019, 6, $1-15$.

9 C. Zhao, Y. Li, W. Zhang, Y. Zheng, X. Lou, B. Yu, J. Chen, Y. Chen, M. Liu and J. Wang, Energy Environ. Sci., 2020, 13, 53-85.

10 A. Bertei, V. Yufit, F. Tariq and N. P. Brandon, J. Power Sources, 2018, 396, 246-256.

11 J. T. S. Irvine, D. Neagu, M. C. Verbraeken, C. Chatzichristodoulou, C. Graves and M. B. Mogensen, Nat. Energy, 2016, 1, 1-13.

12 M. Zubair, R. Song, M. Taqi and S. Lee, Ceram. Int., , DOI:10.1016/j.ceramint.2020.11.002.

13 Z. Cai, Y. Kuru, J. W. Han, Y. Chen and B. Yildiz, J. Am. Chem. Soc., 2011, 133, 17696-17704. 
14 M. Kubicek, Z. Cai, W. Ma, B. Yildiz, H. Hutter and J. Fleig, ACS Nano, 2013, 7, 3276-3286.

15 B. Koo, H. Kwon, Y. Kim, H. G. Seo, J. W. Han and W. Jung, Energy Environ. Sci., 2018, 11, 7177.

16 E. Navickas, Y. Chen, Q. Lu, W. Wallisch, T. M. Huber, J. Bernardi, M. Stöger-Pollach, G. Friedbacher, H. Hutter, B. Yildiz and J. Fleig, ACS Nano, 2017, 11, 11475-11487.

17 Y. Zheng, Y. Li, T. Wu, W. Zhang, J. Zhu, Z. Li, J. Chen, B. Yu, J. Wang and J. Zhang, Nano Energy, 2018, 51, 711-720.

18 M. Choi, I. A. M. Ibrahim, K. Kim, J. Y. Koo, S. J. Kim, J.-W. Son, J. W. Han and W. Lee, ACS Appl. Mater. Interfaces, 2020, 12, 21494-21504.

19 N. Tsvetkov, Q. Lu, L. Sun, E. J. Crumlin and B. Yildiz, Nat. Mater., 2016, 15, 1010-1016.

20 J. Fondard, E. Irisarri, C. Courrèges, M. R. Palacin, A. Ponrouch and R. Dedryvère, J. Electrochem. Soc., 2020, 167, 070526.

21 D. Ding, X. Li, S. Y. Lai, K. Gerdes and M. Liu, Energy Environ. Sci., 2014, 7, 552-575.

22 C. Yun, E. M. Choi, W. Li, X. Sun, T. Maity, R. Wu, J. Jian, S. Xue, S. Cho, H. Wang and J. L. MacManus-Driscoll, Nanoscale, 2020, 12, 9255-9265.

D. Dubbink, G. Koster and G. Rijnders, Sci. Rep., , DOI:10.1038/s41598-018-24025-7.

24 J. Huang, J. L. MacManus-Driscoll and H. Wang, J. Mater. Res., 2017, 32, 4054-4066.

25 E. M. Choi, A. Di Bernardo, B. Zhu, P. Lu, H. Alpern, K. H. L. Zhang, T. Shapira, J. Feighan, X. Sun, J. Robinson, Y. Paltiel, O. Millo, H. Wang, Q. Jia and J. L. MacManus-Driscoll, Sci. Adv., 2019, 5, eaav5532.

26 S. M. Yang, S. Lee, J. Jian, W. Zhang, P. Lu, Q. Jia, H. Wang, T. W. Noh, S. V Kalinin and J. L. 
MacManus-Driscoll, Nat. Commun., 2015, 6, 8588.

27 S. Cho, C. Yun, S. Tappertzhofen, A. Kursumovic, S. Lee, P. Lu, Q. Jia, M. Fan, J. Jian, H. Wang, S. Hofmann and J. L. MacManus-Driscoll, Nat. Commun., 2016, 7, 1-10.

28 S. Cho, J. W. Jang, L. Li, J. Jian, H. Wang and J. L. Macmanus-Driscoll, Chem. Mater., 2016, 28, $3017-3023$.

29 A. Chen, Q. Su, H. Han, E. Enriquez and Q. Jia, Adv. Mater., 2018, 1803241, 1-30.

30 J. Yoon, S. Cho, J.-H. Kim, J. Lee, Z. Bi, A. Serquis, X. Zhang, A. Manthiram and H. Wang, Adv. Funct. Mater., 2009, 19, 3868-3873.

31 W. Ma, J. J. Kim, N. Tsvetkov, T. Daio, Y. Kuru, Z. Cai, Y. Chen, K. Sasaki, H. L. Tuller and B. Yildiz, J. Mater. Chem. A, 2015, 3, 207-219.

32 T. F. Kelly and D. J. Larson, Annu. Rev. Mater. Res., 2012, 42, 1-31.

33 J. Koettgen, S. Grieshammer, P. Hein, B. O. H. Grope, M. Nakayama and M. Martin, Phys. Chem. Chem. Phys., 2018, 20, 14291-14321.

34 M. Bachhav, F. Danoix, B. Hannoyer, J. M. Bassat and R. Danoix, Int. J. Mass Spectrom., 2013, $335,57-60$.

35 T. Kinno, M. Tomita, T. Ohkubo, S. Takeno and K. Hono, Appl. Surf. Sci., 2014, 290, 194-198.

36 R. A. De Souza, J. Zehnpfenning, M. Martin and J. Maier, Solid State Ionics, 2005, 176, $1465-$ 1471.

37 J. Druce, H. Téllez, T. Ishihara and J. A. Kilner, Faraday Discuss., 2015, 182, 271-288.

38 F. Chiabrera, I. Garbayo, L. López-Conesa, G. Martín, A. Ruiz-Caridad, M. Walls, L. RuizGonzález, A. Kordatos, M. Núñez, A. Morata, S. Estradé, A. Chroneos, F. Peiró and A. Tarancón, 
Adv. Mater., 2019, 31, 1805360.

39 Y. Ji, J. A. Kilner and M. F. Carolan, Solid State Ionics, 2005, 176, 937-943.

40 R. E. Usiskin, S. Maruyama, C. J. Kucharczyk, I. Takeuchi and S. M. Haile, J. Mater. Chem. A, 2015, 3, 19330-19345.

41 J. Fleig, H. R. Kim, J. Jamnik and J. Maier, Fuel Cells, 2008, 8, 330-337.

42 J. Maier, Solid State Ionics, 1998, 112, 197-228.

43 F. Shen and K. Lu, Fuel Cells, 2018, 18, 457-465.

44 M. M. Kuklja, E. A. Kotomin, R. Merkle, Y. A. Mastrikov and J. Maier, Phys. Chem. Chem. Phys., 2013, 15, 5443-5471.

45 E. Navickas, T. M. Huber, Y. Chen, W. Hetaba, G. Holzlechner, G. Rupp, M. Stöger-Pollach, G.

Friedbacher, H. Hutter, B. Yildiz and J. Fleig, Phys. Chem. Chem. Phys., 2015, 17, 7659-7669.

46 Z. Shen, S. J. Skinner and J. A. Kilner, Phys. Chem. Chem. Phys., 2019, 21, 13203-13215.

47 W. Jung and H. L. Tuller, Energy Environ. Sci., 2012, 5, 5370-5378.

48 W. Lee, J. W. Han, Y. Chen, Z. Cai and B. Yildiz, J. Am. Chem. Soc., 2013, 135, 7909-7925.

49 M. Kubicek, A. Limbeck, T. Frömling, H. Hutter and J. Fleig, J. Electrochem. Soc., 2011, 158, B727.

50 D. Kim, R. Bliem, F. Hess, J.-J. Gallet and B. Yildiz, J. Am. Ceram. Soc., 2020, 142, 3563.

51 Y. Cao, M. J. Gadre, A. T. Ngo, S. B. Adler and D. D. Morgan, Nat. Commun., , DOI:10.1038/s41467-019-08674-4.

52 M. Pavone, A. B. Muñoz-García, A. M. Ritzmann and E. A. Carter, J. Phys. Chem. C, 2014, 118, 
$13346-13356$.

53 A. A. Ansari, N. Ahmad, M. Alam, S. F. Adil, S. M. Ramay, A. Albadri, A. Ahmad, A. M. AlEnizi, B. F. Alrayes, M. E. Assal and A. A. Alwarthan, Sci. Rep., , DOI:10.1038/s41598-01944118-1.

54 L. Mühlenbein, C. Bhal Singh, A. Hähnel, S. Campbell, C. Hagendorf and A. Bhatnagar, Phys. status solidi, 2020, 1900625.

55 E. Konysheva, S. M. Francis and J. T. S. Irvine, J. Electrochem. Soc., 2010, 157, B159.

56 R. Ramesh and D. G. Schlom, Nat. Rev. Mater., 2019, 4, 257-268.

57 S. J. McCormack and A. Navrotsky, Acta Mater., 2021, 202, 1-21.

58 K. Thompson, D. Lawrence, D. J. Larson, J. D. Olson, T. F. Kelly and B. Gorman, Ultramicroscopy, 2007, 107, 131-139.

59 B. P. Gorman, D. Diercks, N. Salmon, E. Stach, G. Amador and C. Hartfield, Micros. Today, $2008,16,42-47$. 


\section{Figures}
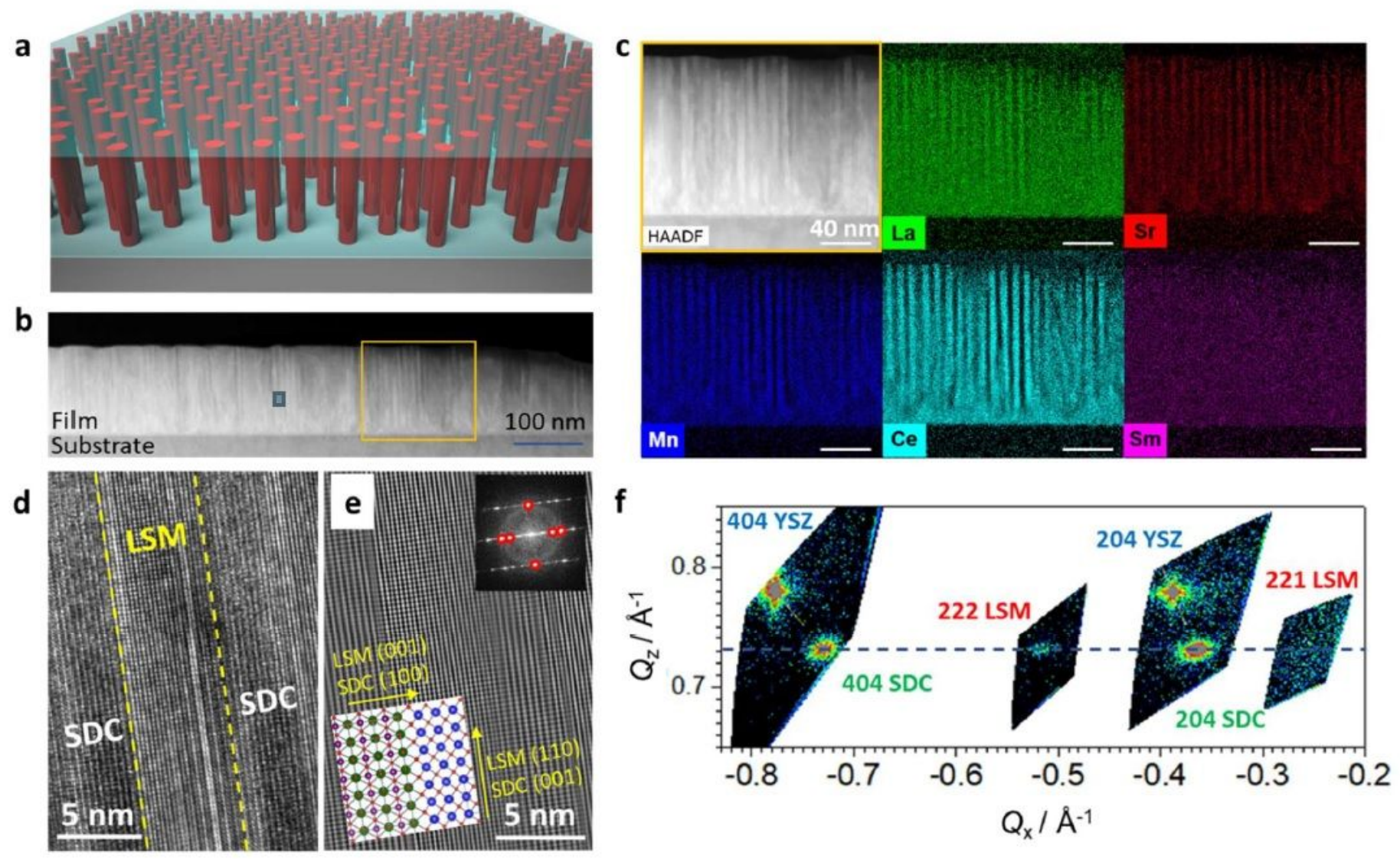

\section{Figure 1}

Structural investigation of LSM-SDC. a) Sketch of the LSM-SDC VAN structure. b) Low magnification image of the film, highlighting the long-range order of the pillar structure. The thickness decrease on the right side should be ascribed to TEM lamella preparation. c) HAADF image and EDS colour maps for a representative columnar region of the film d) HR-TEM of the alternating SDC and LSM columnar structure. e) IFFT of the region highlighted in red in panel c. The atomic ordering and the epitaxial relationship between the LSM (out-of-plane [110], zone axis [1-10]) and the SDC (out-of-plane [001], zone axis [100]) regions is clearly visible, as schematically sketched by the bottom inset. In the inset of top, the original FFT and the filtered reflections (highlighted by the red circles) are shown. f) RSM of the out-ofplane matching 001-SDC ((404) and (204) SDC reflections) and (110)-LSM (222) and (221) reflections) domains. The (404) and (202) substrate reflections are also visible. The dotted horizontal line passes through the maxima of the LSM and SDC reflections, highlighting perfect out-of-plane lattice match between the two phases. Please note that the structural investigation was carried out after after thermal ageing $\left(700^{\circ} \mathrm{C}, 100 \mathrm{hrs}\right)$. 

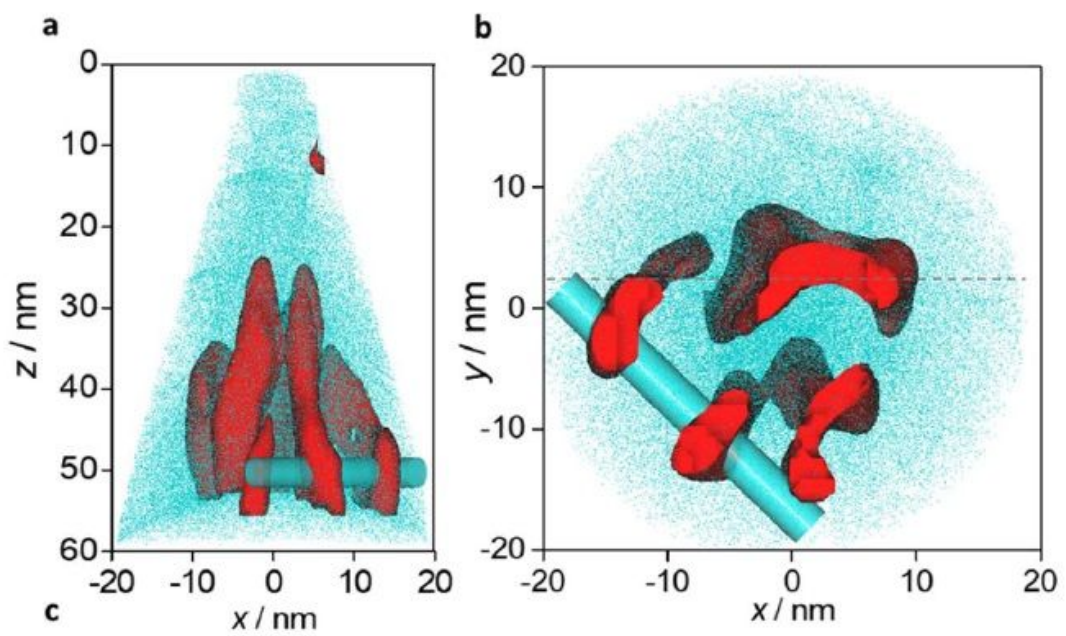

d
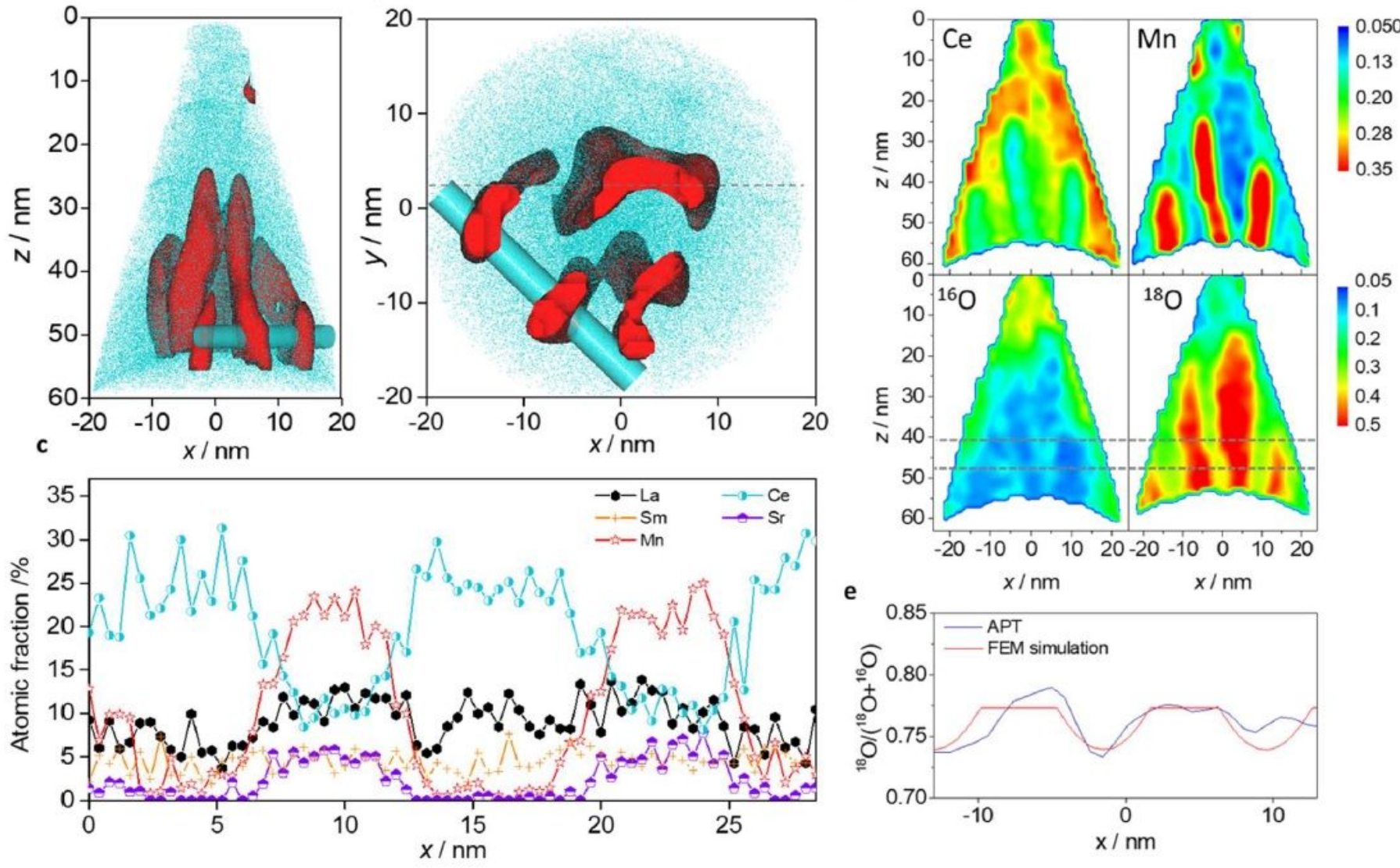

Figure 2

APT on LSM-SDC. Lateral (a) and bottom view (b) 3D renderings, highlighting the areas of LSM (red surface) and SDC (light blue dots) using a 30 at\% La+Sr+Mn isoconcentration surface. c) Cationic atomic fraction along the linescan indicated by the blue cylinder in panels (a) and (b). d) Concentration color maps for Ce, Mn, 180 and 160 from the slice indicated by the grey dotted line in panel (b). e) Experimental (blue - obtained upon integration of the area between the dotted lines in panel d) and simulated (red) 180 fraction profiles. 

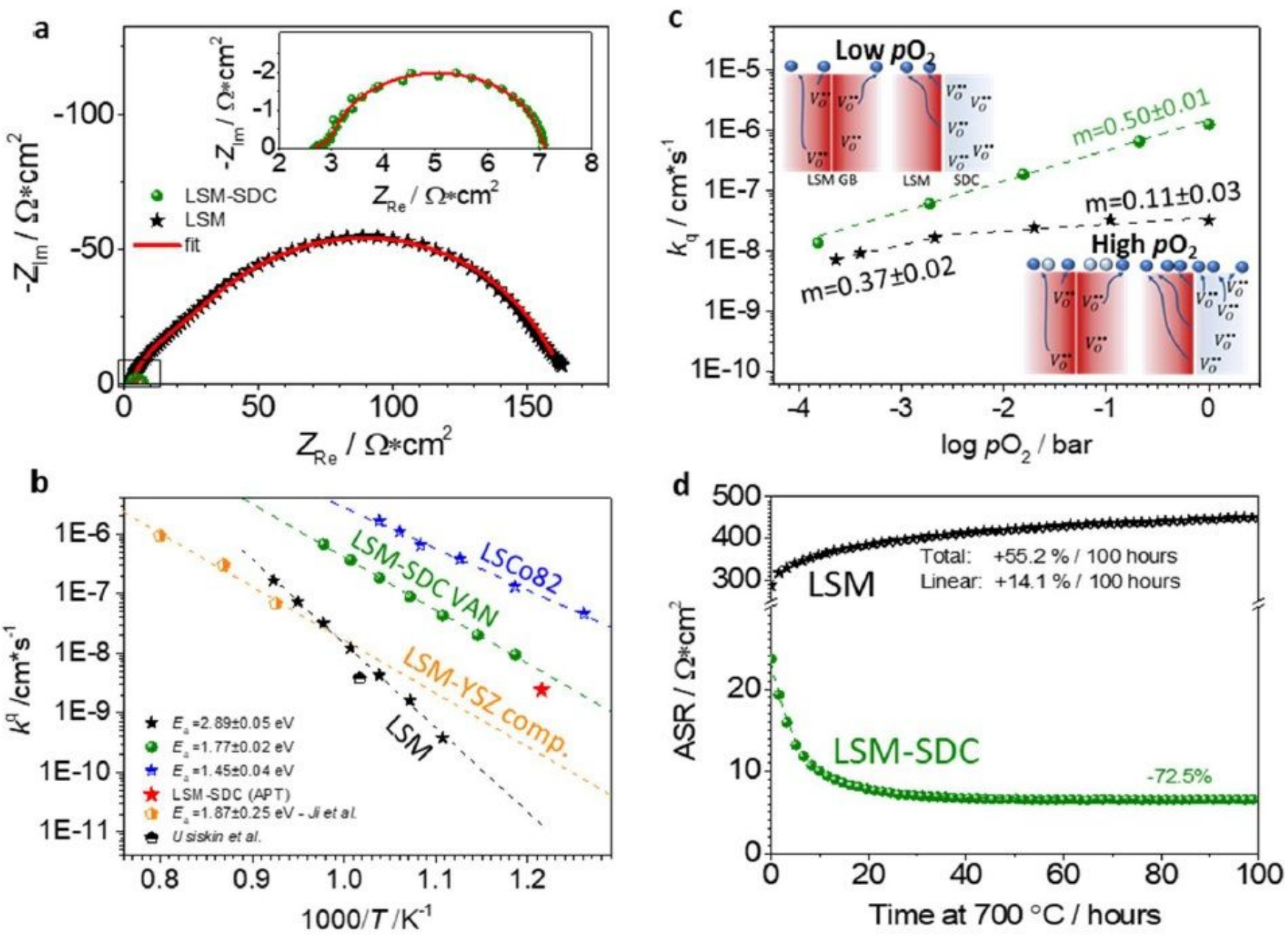

\section{Figure 3}

Electrochemical performance of LSM (black stars) and LSM-SDC (green bullets) nanocomposites. a) Representative EIS Nyquist plots measured at $750{ }^{\circ} \mathrm{C}$ (air atmosphere) in an out-ofplane configuration and using a porous Ag layer as a low impedance counter-electrode for LSM and LSM-SDC. The inset shows the LSM-SDC impedance arc on a smaller scale. b) Surface exchange coefficient kq as a function of inverse temperature, as compared to stoichiometric LSM (from our labs and from Ref. 40), state-of-theart LSM-YSZ dense composites (from Ref. 39) and to state-of-the-art La0.8Sr0.2CoO3 (our labs). The red star represents the value resulting from the FEM simulation of the APT 180 fraction profile. c) p02 dependence for $\mathrm{kq}$. The slopes for LSM and LSM-SDC are indicated. d) ASR vs time for LSM and LSMSDC, measured during ageing treatment. The lines are intended as a guide to the eye. 


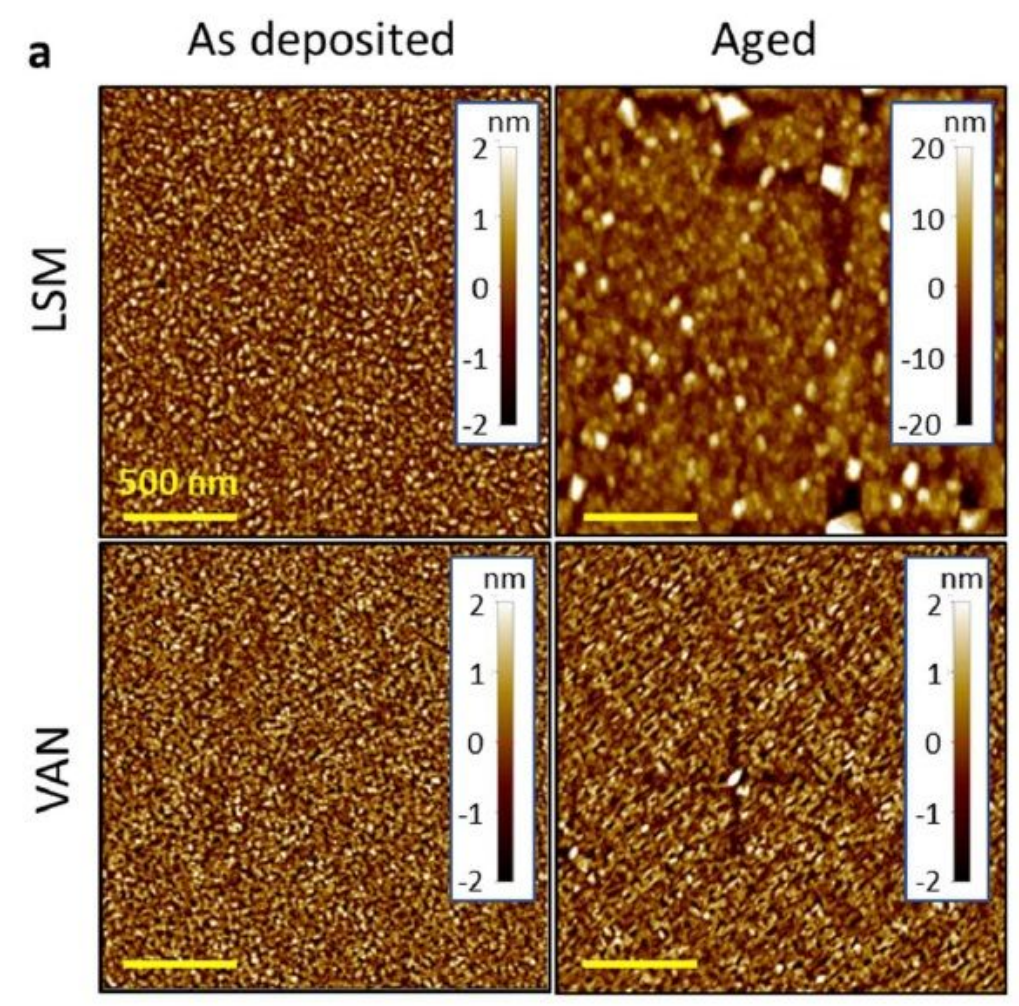

b

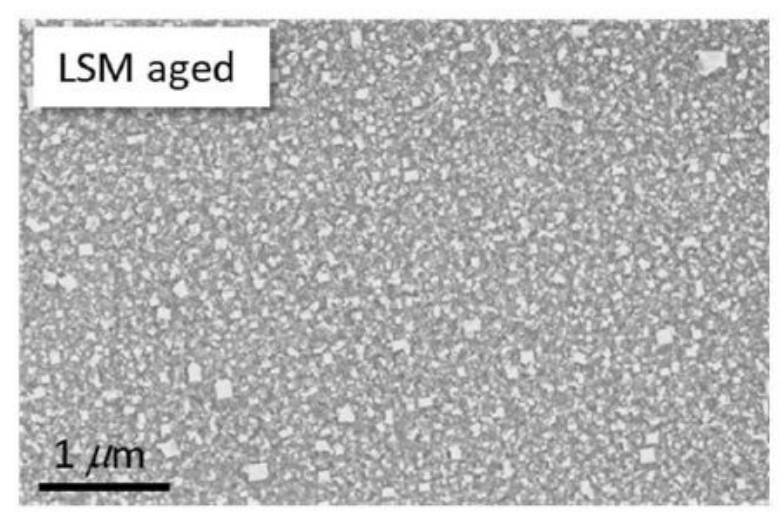

C

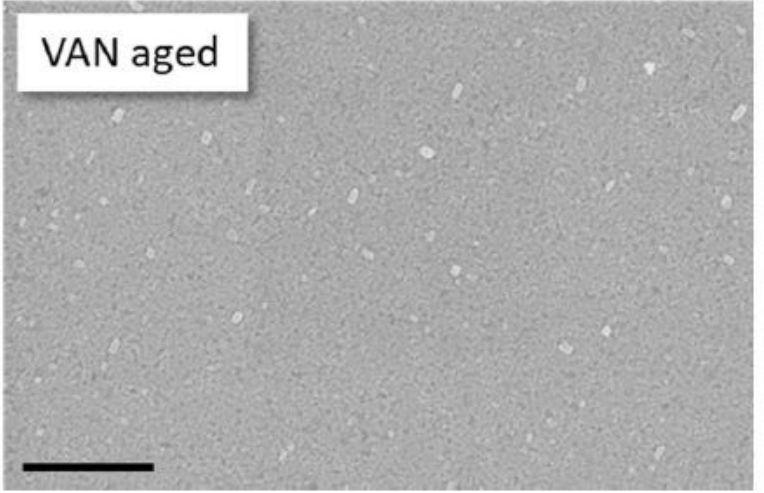

Figure 4

AFM and SEM top-view micrographs for LSM and LSM-SDC. In (a), a full comparison between the surfaces of the two compounds in the as-grown and aged states is provided. The resulting root mean square roughness are: $\mathrm{Rms}=0.8(7.1)$ and Rms $=0.7(1.0) \mathrm{nm}$ for LSM-SDC and single phase LSM as grown (after ageing), respectively. In panels (b-LSM) and (c - VAN), lower magnification SEM for the surfaces after thermal treatment (In-Lens detector $-3 \mathrm{kV}$ acceleration voltage). 

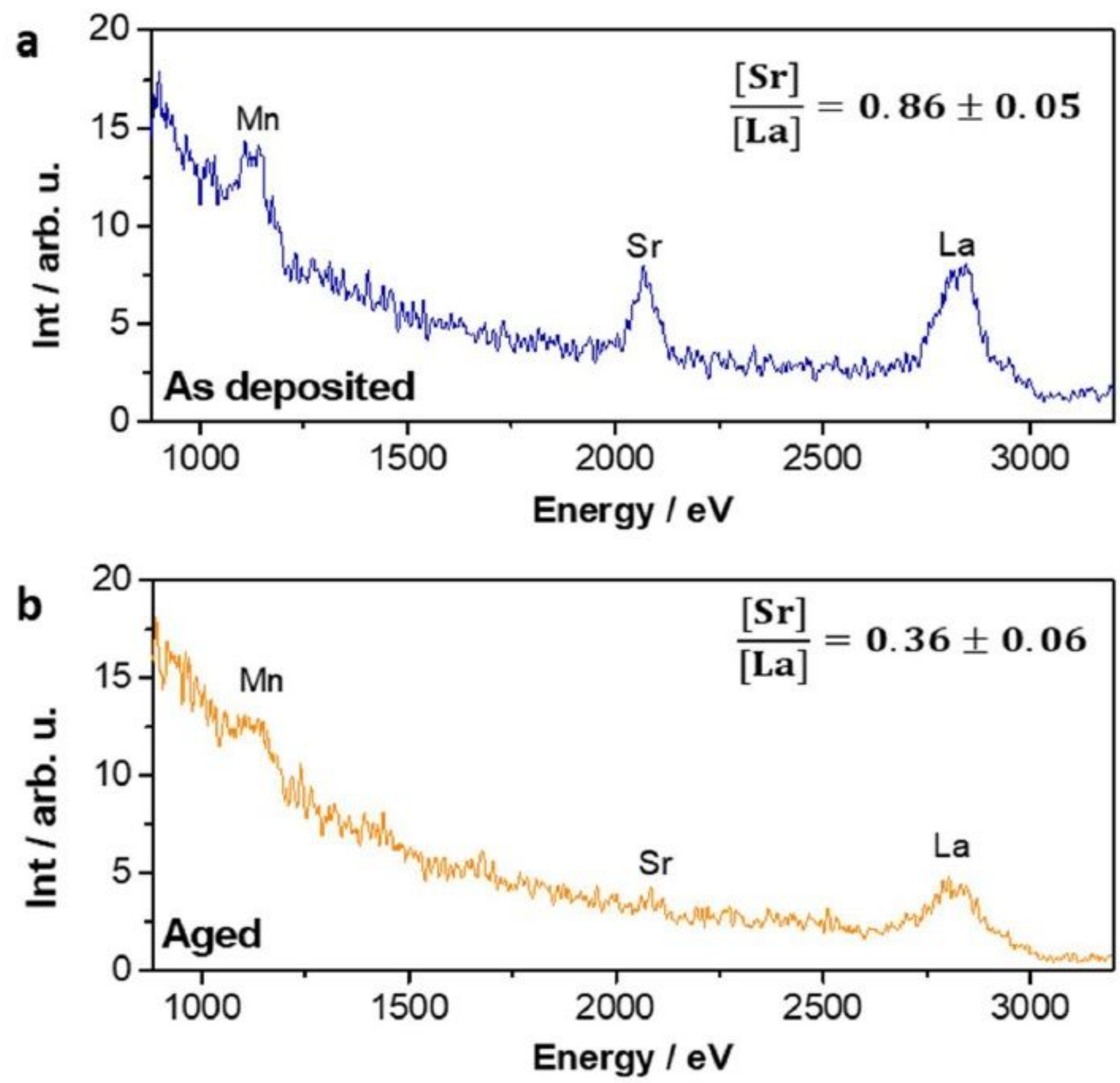

Figure 5

LEIS spectra for LSM-SDC VAN surface as deposited (a) and after thermal treatment $\left(100 \mathrm{hrs}, 700^{\circ} \mathrm{C}-\mathrm{b}\right)$. The stoichiometric ratio is obtained from integration of the peak areas. 


\section{a}

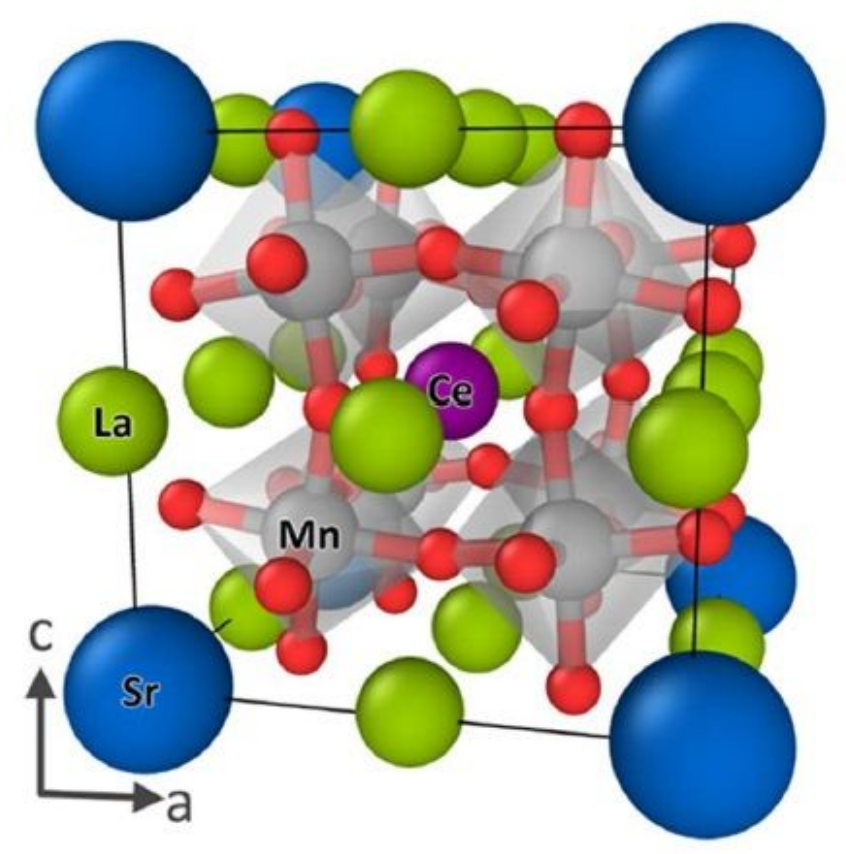

b

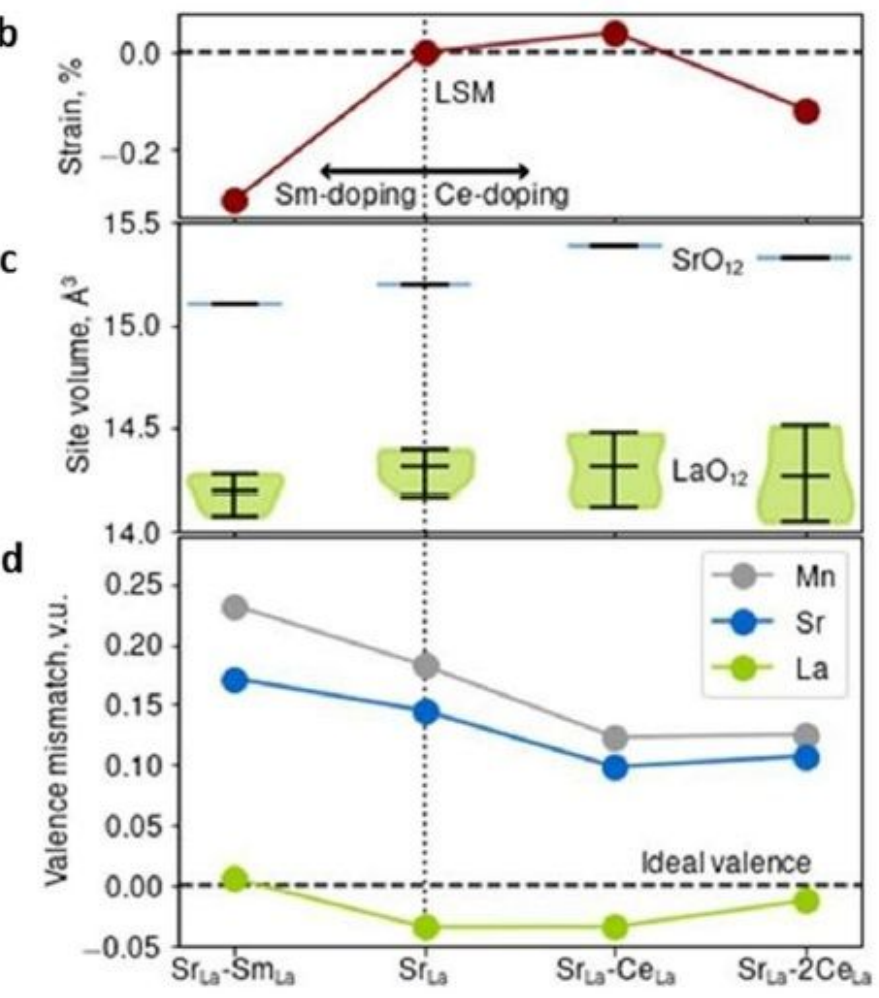

Figure 6

(a) Example of a 40-atom periodic supercell used in DFT calculations showing relaxed positions of the Mn (grey), Sr (blue), La (green), O (red) and Ce (purple) ion and (b) calculated values of the lattice strain due to chemical doping, (c) a box plot showing the mean and distribution of site volumes around the $\mathrm{Sr}$ (blue) and La (green) sites and (d) bond valence mismatch at the ion positions for the different cation dopants, where for example SrLa-SmLa corresponds to a stoichiometry of La0.75Sr0.125Sm0.125MnO3.

\section{Supplementary Files}

This is a list of supplementary files associated with this preprint. Click to download.

- SupplementaryNatComm.pdf 